\title{
How activation, entanglement, and searching a semantic network contribute to event memory
}

\author{
Douglas L. Nelson • Kirsty Kitto • David Galea • \\ Cathy L. McEvoy $\cdot$ Peter D. Bruza
}

Published online: 4 May 2013

(C) Psychonomic Society, Inc. 2013

\begin{abstract}
Free-association norms indicate that words are organized into semantic/associative neighborhoods within a larger network of words and links that bind the net together. We present evidence indicating that memory for a recent word event can depend on implicitly and simultaneously activating related words in its neighborhood. Processing a word during encoding primes its network representation as a function of the density of the links in its neighborhood. Such priming increases recall and recognition and can have longlasting effects when the word is processed in working memory. Evidence for this phenomenon is reviewed in extralistcuing, primed free-association, intralist-cuing, and singleitem recognition tasks. The findings also show that when a related word is presented in order to cue the recall of a studied word, the cue activates the target in an array of related words that distract and reduce the probability of the target's selection. The activation of the semantic network produces priming benefits during encoding, and search costs during retrieval. In extralist cuing, recall is a negative function of cue-to-distractor strength, and a positive function of neighborhood density, cue-to-target strength, and target-tocue strength. We show how these four measures derived from the network can be combined and used to predict memory performance. These measures play different roles in different tasks, indicating that the contribution of the semantic network varies with the context provided by the task.
\end{abstract}

D. L. Nelson $(\bowtie)$

Department of Psychology, University of South Florida,

Tampa, FL 33620, USA

e-mail: dnelson@usf.edu

K. Kitto $・$ D. Galea $・$ P. D. Bruza

Queensland University of Technology, Brisbane,

Queensland, Australia

C. L. McEvoy

School of Aging Studies, University of South Florida, Tampa, FL, USA
Finally, we evaluate spreading-activation and quantum-like entanglement explanations for the priming effects produced by neighborhood density.

Keywords Activation - Quantum-like entanglement . Semantic networks $\cdot$ Semantic memory $\cdot$ Working memory . Priming $\cdot$ Extralist cuing $\cdot$ Word recognition $\cdot$ Reminding

Navigating a complex world requires many kinds of knowledge, and we rely on fast, efficient access to information while engaging hundreds of mental operations, including remembering, thinking, reading, driving, and so on. The brain seems to be wired to respond automatically to cues in its environment by simultaneously activating neighborhoods of related knowledge. We see a friend and her name comes readily, along with related information; we hear the word Atom, and automatically electron, neutron, and bomb are activated. In each instance, related word knowledge is activated, but most of it remains in the shadow of awareness, available but not apparent. Later, some cue reminds us of a prior event, and activates it along with related knowledge; for instance, suppose that we write a check to the electric company, seal it, and put it on the desk. We get ready for work, rush out, and stop the car at the end of the driveway after noticing the mailbox. Our findings indicate that Check and Envelope remind us of the associated words bill, money, mail, and the Mailbox cue activates links to bill, mail, and mailman. Mailbox succeeds as a cue because it implicitly activates words associated with a momentarily forgotten task that reminds us to pay the bill.

In our research, we ask people to study words and then we provide word cues to remind them of what they have studied. We use free association norms to determine what related words are likely to be activated when a word is experienced, and in this article, we will describe how free association can be used to measure the probabilities of links between words, and how such links reveal a large and 
organized associative/semantic network of words. Our motivating thesis is that the brain stores and retrieves experience with objects and events, and words are invented to represent such information. As experiences and the words that represent them are repeated, they link together in interconnected semantic neighborhoods. Such neighborhoods differ in both size and connective density, with some neighborhoods being so dense that they generate category names such as fruit, or "topics" such as alcohol or drug abuse (Goldman, 1999; Griffiths, Steyvers, \& Tenenbaum, 2007; Stacy, 1997).

One purpose of this article is to show how the semantic network contributes to episodic memory for recently experienced word events. The findings show that experiencing a familiar word during a study episode activates related words that prime its activation state in this network and make it easier to recall and recognize. We will review evidence from data pooled over many experiments suggesting that cognitive processes operate on this network, and we will evaluate equations based on measures taken from the network in order to predict recall in the presence of a related cue. We will show how the effects of word knowledge change during encoding and retrieval, and with test delay and semantic context, and we will discuss how priming effects related to automatic activation can persist over long intervals. As a second purpose of the article, we address the issue of how network activation works when a word is processed during study. We will challenge the spreading-activation explanation for priming effects and suggest that experiencing a word simultaneously activates links that encompass the word's local neighborhood. Finally, we will present a quantum-like entanglement model that describes and predicts how parallel activation captures neighborhood density and the resulting priming effects.

\section{The semantic network}

Semantic/associative networks (shortened to semantic in this article) can be constructed by presenting lists of words to subjects asked to produce the first word to come to mind that is meaningfully or associatively related (e.g., Cramer, 1968; Deese, 1965; De Deyne \& Storms, 2008a, 2008b; Nelson, Dyrdal, \& Goodmon, 2005; Nelson, McEvoy, \& Schreiber, 2004). Free association measures what one group of brains knows about semantic relationships and predicts what other groups of brains will be likely to know. It is a direct way to measure what related words are likely to come to mind when a word is experienced, and the validity of such measures depends on how well they predict performance in word-processing tasks.

Free association to a word produces an array of associated words that vary in probability and number. First-response associates are highly reliable $(r=.89)$, even for responses produced by only two subjects, and we assume that they index a word's nearest neighbors (Nelson, McEvoy, \& Dennis, 2000).
We collected such norms on 5,000 words over more than 20 years using more than 6,000 subjects, with an average of about 150 subjects responding to each word. The words were normed for addressing specific research questions that evolved over time, and initially, we collected norms to study the effects of cue-target relationships in the extralist-cuing task. In this task, subjects study a list of unrelated words that are not directly associated to one another, called targets $(T)$, and the recall of each target is prompted during testing by a semantically related word cue $(Q)$. Early interest focused on the effects of two freeassociation measures, forward cue-to-target strength and backward target-to-cue strength (see Table 1 for abbreviations and calculations of these and other variables). The experimental findings showed that extralist cued recall increases as a positive function of each link (e.g., Nelson, Fisher, \& Akirmak, 2007): Recall is more likely when a cue strongly activates a target in free association, and in addition, an associate that is strongly activated by the target is a more effective cue during test than is a weakly activated associate.

Other findings showed that words vary in terms of how many different associates they produce in free association, and that the number of response alternatives produced by a word that is used as a test cue has a robust but negative effect on recall (called the cue set size effect in our experiments): The more associates a cue has, the less the chance of recovering one of its associates that has been studied as the target. This finding generalizes across word types, taxonomic category names, word stems, rhymes, and pictures (Nelson \& Castano, 1984; Nelson \& McEvoy, 1979; Nelson, Walling, \& McEvoy, 1979). Cue set size, however, is an inclusive measure that includes the target, indirect facilitating links that join the cue and target (mediators and shared associates), and alternative responses. For this article, we created a relative index that separates the alternative responses responsible for negative effects from the target and indirect links in statistical analyses of the data from the earlier experiments. Alternative responses are now called distractors, and as is indicated in Table 1, they are computed as proportions that measure relative distractor strength; the probability of selecting a distractor instead of the target increases as distractor strength increases.

Cue set size findings created a need to norm more words, and this task became routine after learning that studied targets with more links among their associates are more likely to be recalled and recognized (e.g., Nelson, Bennett, Gee, Schreiber, \& McKinney, 1993; Nelson, McKinney, Gee, \& Janczura 1998; Nelson, Zhang, \& McKinney, 2001). This phenomenon was called connectivity in these experiments, but to be more consistent with the literature on related topics, we will refer to it in Fig. 1 and elsewhere as neighborhood density. Measuring neighborhood density required us to norm a word in order to identify its closest associates, and then to norm each of its associates using independent samples of subjects. Figure 1a shows a toy network for word $T$ that 
Table 1 Variables shown to affect extralist cued recall, along with their computations

\begin{tabular}{ll}
\hline Variables & Computation \\
\hline Cue-to-target strength, $S_{q t}$ & \# subjects producing target in free association $\div$ sample size \\
Target-to-cue strength, $S_{t q}$ & \# subjects producing cue in free association $\div$ sample size \\
Cue-to-distractor strength, $S_{q d}$ & \# cue distractors $\div$ \# cue associates \\
Primed target strength, $S_{t^{\prime}}$ & \# links among target's associates $\div$ \# possible links \\
Mediator strength, $S_{m}$ & \# mediators $\div$ \# cue associates \\
Shared-associate strength, $S_{s a}$ & \# shared associates $\div$ \# target associates \\
Associate-to-target strength, $S_{a t}$ & \# associate-to-target links $\div$ \# target associates \\
Target-to-distractor strength, $S_{t d}$ & \# target distractors $\div$ \# target associates
\end{tabular}

* Distractors exclude the target, mediators, and shared associates. Mediators provide indirect links from the test cue to the target (e.g., Proton $\rightarrow$ electron $\rightarrow$ Atom). Shared associates occur when the cue and target have the same associate in common (e.g., Proton $\rightarrow$ electron and Atom $\rightarrow$ electron).

produces three directly related associates, along with the four links that connect them. Figure $1 \mathrm{~b}$ illustrates this network as an $n \times n$ asymmetrical adjacency matrix for word $T$ that represents its neighborhood. Placing $T$ s s closest associates $(A)$ on the columns and again on the rows defines its neighborhood in matrix form. Each of $T$ 's associates is normed separately, and we measure whether or not $\{1,0\}$ a link connects these associates - for instance, reading along the rows, $A_{12}=1$ and $A_{13}=1$, indicating that $A_{1}$ is linked to both $A_{2}$ and $A_{3}$. Figure $1 \mathrm{c}$ calculates neighborhood density by summing the number of associate-to-associate links in the matrix and dividing by the number of possible links. As with cue-to-target, target-to-cue, and distractor strengths, neighborhood density is a relative index of strength that varies from 0.0 to 1.0 . Density increases as the number of links among the target's associates increase relative to the size of its neighborhood. Neighborhood density theoretically measures primed target strength, on the assumption that targets with higher neighborhood densities are primed to higher activation levels within the semantic network. For example, Trombone has 85 out of 240 possible links among its associates, whereas Basket has 10 out of a possible 342 links. Their respective primed strengths are .354 and .029 , and the findings show that, with other variables controlled, words such as Trombone are more likely to be recalled and recognized than are words like Basket. In the last section of this article, we will focus on neighborhood density in predicting recall and
Fig. 1 Measuring primed target strength on the basis of neighborhood density a. Graph depicting Word T, its 3 associates, and their 4 connecting links

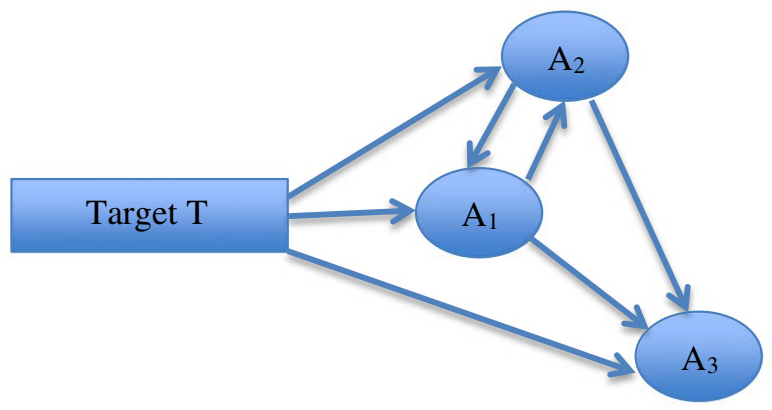

b. Asymmetric adjacency matrix depicting links among T's associates

Normed Associates of T

\begin{tabular}{|l|l|c|c|c|}
\hline & Target $\mathrm{T}$ & $\mathrm{A}_{1}$ & $\mathrm{~A}_{2}$ & $\mathrm{~A}_{3}$ \\
\hline Associates & $\mathrm{A}_{1}$ & --- & 1 & 1 \\
\hline Normed & $\mathrm{A}_{2}$ & 1 & --- & 1 \\
\hline As Cues & $\mathrm{A}_{3}$ & 0 & 0 & --- \\
\hline
\end{tabular}

c. Primed target strength, $S_{t^{\prime}}$

$$
S_{t^{\prime}}=\frac{\sum_{i=1}^{n} \sum_{j=1}^{n} A_{i j}}{n(n-1)}=\frac{4}{3(3-1)}=.667
$$


recognition and propose a quantum-like model for explaining its effects.

What does free association measure?

In free association, response production is not really "free," in the sense that any response is always acceptable. Free association is restricted because the instructions bias responses, from the very general - any word - to the very specific - category instances, properties, rhymes, feelings, topics like "alcohol makes me feel," and so on (e.g., Battig \& Montague, 1969; Cramer, 1968; MacRae, Seidenberg, \& McNorgen, 2005; Reich \& Goldman, 2005). Because the task is so familiar, individuals can access and generate any type of related word on the basis of instructions that set a comprehensible goal that defines a context for eligible words. Different biases produce different semantic maps, and at this time, no single "best" map predicts the effects of all types of mental operations on all types of word knowledge (Maki \& Buchanan, 2008). For our norms, we requested a response that reflects meaning or association, which allowed responses to be based on a variety of semantic relationships.

The second word in the task name, association, is also misleading, because it implies that cue-to-target strength is determined only by an association formed by coexperiencing words $Q$ and $T$ in the same context. Association by learning is critical, but observing the existence of a link reveals nothing about its source. Free association indicates the probability that one word will lead to production of another and tells us nothing about the information used to produce the response. The observation could be generated through learned association, recency, imagery, semantic similarity, or contrast. Several studies have shown that semantic information concerning categories, properties, emotions, and so on, is well represented in free-association data (Borge-Holthoefer \& Arenas, 2010; Brainerd, Yang, Reyna, Howe, \& Mills, 2008; De Deyne \& Storms, 2008a; Hutchison, 2003; Monaco, Abbott, \& Kahana, 2008; Steyvers, Shiffrin, \& Nelson, 2005). Most importantly, when we refer to the "semantic network," we are referring to the normalized network that emerges from our free-association procedure. Furthermore, we assume that every person has a variant of such a network in memory and that processes such as activation and search operate directly on this representation.

Organization of the semantic network

In many sciences, networks tend to be large, asymmetric, and irregular structures that are well suited for describing complex systems (Newman, 2003). Networks describe links between people (Moreno, 1934), computers (Barabási, 2002), neurons (Watts \& Strogatz, 1998), metabolic states (Jeong, Tombor, Albert, Oltvai, \& Barabási, 2000), and words (Steyvers \&
Tenenbaum, 2005). Their topological structure helps scientists understand how different kinds of entities communicate in interactive systems, and they have been used to describe a wide range of processes, including percolation, spread of disease, phase transitions, protein interactions, and computer search, as well as activation and search (Barabási, 2002; Nelson et al., 1998; Newman, 2003). Networks are also useful to scholars investigating complexity itself (e.g., Barabási, 2002; Palla, Derényi, Farkas, \& Vicsek, 2005; Steyvers \& Tenenbaum, 2005; Strogatz, 2003; Watts \& Strogatz, 1998).

Research across disciplines has indicated that different networks are organized in strikingly similar ways. By treating networks as graphs and borrowing the terminology and mathematics of graph theory, researchers have shown that networks built from different entities and types of links tend to have similar statistical profiles. Of particular interest, Steyvers and Tenenbaum (2005) used our norms to create a semantic network by generating a 5,018 $\times 5,018$ word matrix in which normed cues were represented on the rows and responses were shown on the columns, as in Fig. 1b. Their analysis shows that the network is sparsely linked, with each word being directly connected to an average of only 12.7 other words. Furthermore, short path links are characteristic of all smallworld networks (Watts \& Strogatz, 1998). The average directed path length from any one word in our norms to any other takes only 4.27 links or "steps," so the semantic network qualifies as a small-world network. More importantly for this article, a property termed clustering in graph theory is described in this article by neighborhood density, the relative number of links among a word's associates (Fig. 1c).

The network analyses indicated that words in the semantic network are directly connected to only a few other words, but on average, they are connected to nearly all other words in the net within a few mediating links. The network has a smallworld structure composed of words with varying levels of neighborhood density that are linked by short paths to and from other words. As a network, words are represented as a system of nodes and links, as opposed to isolated pairs, and theoretically, a mental process operating on one word in the system has the potential to change the states of related words in the network. The system in which words reside is distributed, but many alternative paths can lead to any single word, and short paths provide shortcuts that may affect the activation states of distant concepts (Watts \& Strogatz, 1998).

We assume that the semantic network is a dynamically evolving, complex system. Complexity arises because we know thousands of words that are linked in semantic memory in ways that can affect how well a given word will be remembered. Dynamics arise because portions of the system are always growing or weakening in response to experience (Hills, Maouene, Riordan, \& Smith, 2010; Steyvers \& Tennenbaum, 2005). The network is also dynamic in terms of how it responds to the contexts provided by different 
tasks. We next review findings showing that a target's neighborhood density has different effects, depending on the task and the semantic context in which it is experienced.

\section{Knowledge $\times$ Context interactions}

In this section, we review how the semantic network is engaged in a variety of tasks, including cued recall, primed free association, intralist cuing, and word recognition. We initially focus on the extralist-cuing task, resembling the mailbox example above, because of its importance to everyday memory events and because it is more interesting from a network perspective. Pooling the results over dozens of extralist-cuing experiments provides a database on thousands of word pairs that we will use to identify the most important network variables and how they can be incorporated into an equation that effectively predicts recall.

\section{Extralist cuing}

During the study phase of the standard extralist task, a list of unrelated familiar words is presented one at a time for $3 \mathrm{~s}$, and subjects read each word aloud and attempt to remember as many as possible. Test instructions are provided immediately after the last study word. During testing, another set of words is presented one at a time at a self-paced rate, and the instructions ask subjects to use each cue to recall a related target word from the study list (e.g., use the cue "Proton" to recall the target "Atom"). The procedure is called extralist because the test cues are physically unavailable during study, and hence, successful recall depends on the cue accessing the target in the semantic network. This characteristic makes extralist cuing an ideal "reminding" paradigm for studying how prior knowledge captured by a single word can be used to respond to the environment.

Table 1 presents a list of eight variables shown to affect recall in extralist cuing that have been incorporated into a statistical prediction algorithm called Pier2, standing for "processing implicit and explicit representations" (see, e.g., Nelson, Goodmon, \& Ceo, 2007, and Nelson \& Zhang, 2000 , for description and computational examples). One purpose of this article is to report a simplified computation that grew out of two problems with Pier2: It overpredicts recall, and the eight variables in its prediction equation are not on the same scale, with some representing strengths, such as cue-totarget strength, and others representing sums of strengths. Although the equation is well correlated with recall, we thought that transforming each variable to the same scale would solve the over prediction problem. Just as importantly, given correlations among the variables, not all of them may be necessary for predicting recall thus simplifying the problem of incorporating them into a prediction equation. As we will show, transforming our past measures into proportions unexpectedly forced us to reconceptualize Pier and to revise our interpretation of both cue and target set size effects.

All eight variables shown in Table 1 were transformed to free-association-determined strengths that ranged from 0.0 to 1.0 and were entered into an extralist-cuing database composed of 4,068 cue-target pairs rescored at the item level. ${ }^{1}$ In the articles that reported the original experiments, the data were pooled over items, and the usual subject-based analysisof-variance procedures were applied to determine what variables were significant. In contrast, in this article, the data are pooled over subjects in all analyses. The number of subjects who correctly recalled a target relative to the total number who studied the target computes the probability of recalling each cue-target pair (see Nelson \& Zhang, 2000). Both subject and item analyses indicate whether a variable is important, but item analyses are critical for predicting the probability of recalling a given cue-target pairing. This type of analysis allowed us to determine the influence of a variable over thousands of cue-target pairs, which is important when the goal is to determine how and how much the network contributes to memory for recent word events. In addition, the item analysis allowed us to use regression analyses that statistically controlled for correlations among the variables.

In the database, the cue-target pairs were shown in two columns, with other columns representing the observed and predicted probabilities of correct recall, information on how the list was studied, measures of the eight variables, and other related information. The analyses were restricted to 2,803 pairs studied in the standard task, and a preliminary analysis was used to determine whether the new scaling procedure eliminated some variables when correlations with other variables were statistically controlled in a simultaneous multiple regression. Probability of correct recall was the dependent measure, and measures of the eight variables served as predictors.

The preliminary analysis results indicated that the first four variables shown in Table 2 were more important for predictive purposes. The regression was significant $[F(8,2794)=$ 205.30, $\left.M S_{\text {res }}=.042, R=.61, R^{2}=.37\right]$, and the partial correlations shown in the bottom row of Table 2 indicate that the variables represented in the first four columns were more strongly correlated with recall than were the last four. The four variables having the largest effects were cue-to-target strength, $S_{q t}$, target-to-cue strength, $S_{t q}$, cue-to-distractor strength, $S_{q d}$, and neighborhood density as a measure of primed target strength, $S_{t^{\prime}}$. Of the last four variables, shared associate strength and associate-to-target strength failed to have significant effects in this analysis, and mediator strength and targetdistractor strength had reduced effects because of correlations

\footnotetext{
${ }^{1}$ All databases mentioned in this article are available on request from the first author.
} 
Table 2 Correlations (above diagonal) and partial correlations (below diagonal) among the eight network variables that affect extralist cued recall

\begin{tabular}{lcccccccccc}
\hline & $S_{q t}$ & $S_{t q}$ & $S_{q d}$ & $S_{t^{\prime}}$ & $S_{m}$ & $S_{s a}$ & $S_{a t}$ & $S_{t d}$ & $r$ With Prob. Recall \\
\hline$S_{q t}$ & - & -.01 & -.29 & -.04 & .27 & .04 & .17 & -.02 & .46 \\
$S_{t q}$ & .01 & - & .11 & .20 & -.20 & .18 & -.21 & -.30 & .19 \\
$S_{q d}$ & -.13 & .13 & - & -.16 & -.73 & -.43 & -.11 & .37 & -.37 \\
$S_{t^{\prime}}$ & -.10 & .08 & .07 & - & .08 & .63 & .10 & -.58 & .26 \\
$S_{m}$ & -.02 & .04 & -.70 & -.07 & - & .23 & .45 & -.15 & .30 \\
$S_{s a}$ & .00 & -.13 & -.15 & .34 & .02 & - & -.04 & -.87 & .34 \\
$S_{a t}$ & .11 & -.14 & .31 & .22 & .51 & -.02 & - & .11 & .10 \\
$S_{t d}$ & .06 & -.25 & .08 & -.06 & .05 & -.76 & .08 & -.00 \\
Partial $r$ With Prob. Recall & .42 & .19 & -.10 & .11 & .06 & .02 & .02 & -.07 \\
\hline
\end{tabular}

with other measures. These four variables were dropped from the prediction equations that will be described later, because they were redundant and reduced predictability when included. The failure of associate-to-target strength to affect recall is important to spreading-activation theory, and we will return to this result later. The failure of target-distractor strength to achieve significance deserves special notice, because this variable played a key role in our earliest investigations of the semantic network. Many experiments had focused on the effects of varying target set size because it has robust negative effects on cued recall, but as with cue set size, it is correlated with several variables that have positive effects (see Table 2). However, we are not dismissing target set-size effects as irrelevant. In the conversion to proportions, target set size determined the size of the associative matrix, and hence the relative magnitude of neighborhood density. Given a fixed number of links among the target's associates, neighborhood density decreases as target set size increases, so the negative effects of target set size are built into the density index.

The first row of Table 3 shows that the results of a second analysis, limited to the four most important predictors of recall, are significant overall, and that each predictor has significant effects. The correlations, partials, and descriptive statistics are shown in Table 4. There, the partial correlations are shown in the bottom row, and each variable is significantly correlated with recall after correlations among the variables are statistically controlled. Of the variance that could be explained, a separate, unforced stepwise regression showed a highest-to-lowest ordering of cue-to-target strength $S_{q t}=58.9 \%$, neighborhood density $S_{t^{\prime}}=20.8 \%$, cue-todistractor strength $S_{q d}=11.8 \%$, and target-to-cue strength $S_{t q}=8.5 \%$. Recalling the target depends most on the cue-totarget strength $S_{q t}$, and next-most on neighborhood density $S_{t^{\prime}}$. Target-to-cue strength $S_{q t}$ has a smaller positive effect, and

Table 3 Results of regression analyses evaluating the effects of the four predictors as a function of memory task

\begin{tabular}{|c|c|c|c|c|c|c|c|c|c|}
\hline & $F$ & $M S_{\text {res }}$ & $R$ & Adj $R^{2}$ & $S_{q t}$ & $S_{t q}$ & $S_{q d}$ & $S_{t^{\prime}}$ & Study Cond. \\
\hline Extralist Cuing & $F(4,2803)=387.36$ & .043 & .597 & .355 & & & & & \\
\hline Std. Coeff. & & & & & .395 & .183 & -.246 & .194 & - \\
\hline$S E$ & & & & & .034 & .028 & .022 & .034 & - \\
\hline$t$ Value & & & & & 24.81 & 11.64 & 15.08 & 12.26 & - \\
\hline Primed Free Assoc. & $F(5,464)=47.42$ & .019 & .583 & .332 & & & & & \\
\hline Std. Coeff. & & & & & .441 & .162 & -.225 & .081 & .145 \\
\hline$S E$ & & & & & .100 & .069 & .037 & .087 & .007 \\
\hline$t$ Value & & & & & 10.77 & 4.19 & -5.18 & 2.07 & 3.76 \\
\hline Intralist Cuing & $F(4,509)=13.17$ & .037 & .306 & .087 & & & & & \\
\hline Std. Coeff. & & & & & .263 & .011 & -.090 & -.056 & - \\
\hline$S E$ & & & & & .085 & .052 & .049 & .001 & - \\
\hline$t$ Value & & & & & 5.78 & $.246^{\dagger}$ & $-1.91^{\dagger}$ & $-1.24^{\dagger}$ & - \\
\hline Recognition & $F(3,1647)=221.02$ & .919 & .536 & .286 & & & & & \\
\hline Std. Coeff. & & & & & - & - & - & .071 & .381 \\
\hline$S E$ & & & & & - & - & - & .143 & .033 \\
\hline$t$ Value & & & & & - & - & - & 3.37 & 18.33 \\
\hline
\end{tabular}

\footnotetext{
${ }^{\dagger}$ Not significant
} 
Table 4 The four strongest predictors of extralist cuing, with correlations (above diagonal) and partial correlations (below) in the first five columns, and descriptive statistics in the last three columns

\begin{tabular}{lllllllll}
\hline & $S_{q t}$ & $S_{t q}$ & $S_{q d}$ & $S_{t^{\prime}}$ & Prob. Recall & Mean & $S D$ & Range \\
\hline$S_{q t}$ & - & -.01 & -.29 & -.04 & .46 & .152 & .120 \\
$S_{t q}$ & -.05 & - & .11 & .20 & .19 & .099 & $.00-.818$ \\
$S_{q d}$ & -.15 & .21 & - & -.16 & -.37 & .605 & .194 \\
$S_{t^{\prime}}$ & -.18 & .17 & -.13 & - & .26 & .152 & $.00-.917$ \\
Prob. Recall & .43 & .22 & -.27 & .23 & - & .518 & $.091-1.00$ \\
\hline
\end{tabular}

only cue-to-distractor strength $S_{q d}$ has negative effects on recall: All things being equal, a cue that activates a higher proportion of distractors is more likely to fail.

\section{Predicting extralist cued recall}

Retrieving a target event from the semantic network can be direct when cue-to-target strength is very strong, but retrieval becomes a problem as the cues become weaker and retrieval less certain. For the most part, in our experiments cue-to-target strength was generally weak (averaging .15) because we were manipulating several variables in factorial designs and needed to keep recall below ceiling. Given the uncertainty of retrieval, a test cue was more likely to utilize

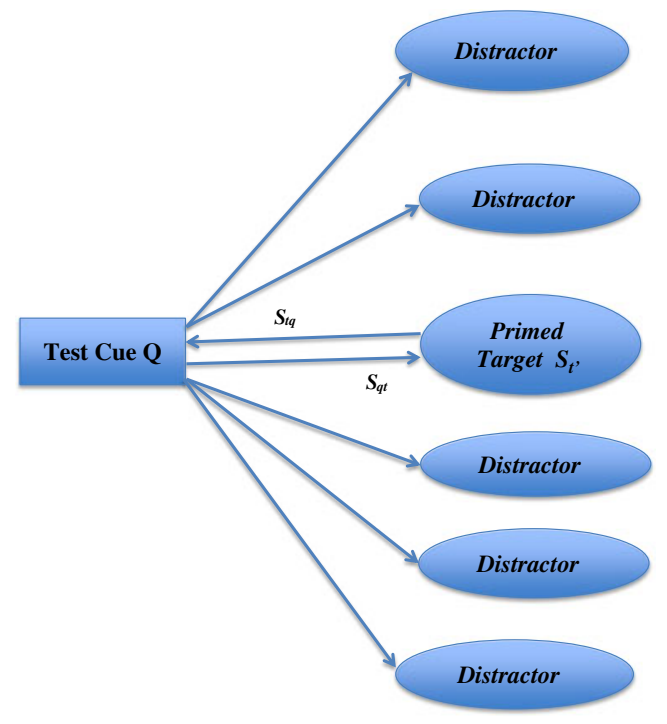

Fig. 2 Recovering primed target $T^{\prime}$ from its test cue $Q$. Cue-to-target strength $\left(S_{q t}\right)$ is represented in the arrow from the test cue to the target, and target-to-cue strength $\left(S_{t q}\right)$ is represented in the arrow from the target to the test cue. Both strengths are taken directly from freeassociation norms. Primed target strength $\left(S_{t^{\prime}}\right)$ is calculated, as an example, in Fig. 1 (see also Eq. 4 in the text). The cue-distractor strength in this figure is determined by dividing the number of distractors by the total number of responses produced to the cue in free association, after eliminating mediators and shared associates. Mediators and shared associates facilitate recall and are not counted as distractors, and because there are none in the figure, the cuedistractor strength is $1 / 6$ or .167 all of the available information. In the Pier2 framework, measures of variables known to affect recall had been combined in a single prediction equation. We updated this equation now because of the changes in the number of key measures and in their underlying scales, and we will refer to the new approach as Pier3. There are many ways to combine four predictors, and we evaluated many plausible computations. The equation that we report below is the best general predictor, and although it does not constitute a model in the same sense as general memory models (e.g., the search of associative memory [SAM] model), it carries implications for how a cue reminds us to recover a recent word event from the semantic network.

Figure 2 illustrates the positive and negative effects involved when a test cue activates its associates, given the goal of recalling a related and recent word event. We assume that the four sources of strength represented in the figure act together in affecting probability of recall. The target activates its associative network, and this activation strengthens the target's representation in semantic memory. By activating an array of related associates, the test cue narrows the range of potential targets, and the target is selected from within this array. Cue-to-target $\left(S_{q t}\right)$ and target-to-cue $\left(S_{t q}\right)$ strengths locate the primed target in the array, and its recall is more likely when these links are stronger and when the target has been primed to a higher level of activation during study $\left(S_{t^{\prime}}\right)$. Theoretically, target priming makes its representation more distinct within the array of associates activated by any related cue and increases the likelihood of selecting the target. In contrast, distractors have negative effects on recall, and cueto-distractor $\left(S_{q d}\right)$ strength is interpreted as sampling error. As distractor strength increases, the probability of sampling a distractor as opposed to the target increases.

Equation 1 brings the four sources of information about the target together. Words in the semantic network exist in a tangle of correlated links loosely organized into a complex system, and recall in extralist cuing is based on more than just how strongly the test cue is related to its target. Equation 1 is a SAM-like equation stating that the semantic network's contribution to target recall in extralist cuing can be predicted by computing the probability of recalling a primed target, given the test cue. $P\left(T^{\prime} / Q\right)$ is a ratio of positive sources of 
information about the target, relative to the total amount of information activated (see, e.g., Raaijmakers \& Shiffrin, 1981). The probability of recalling a primed target is a function of total cue-target strength, relative to the total amount of information activated by the test cue, which includes total cue-target strength and distractor strength. Total cue-target strength $S\left(Q, T^{\prime}\right)$ is captured in Eq. 2 by summing the cue-totarget and target-to-cue strengths and adding the result to the target's neighborhood density. Cue-distractor strength is defined in Eq. 3 as the number of distractors relative to the number of associates activated by the test cue (cue set size). Finally, Eq. 4 measures target priming as a function of its relative neighborhood density.

All of these sources of information are embedded in the semantic network, and we assume that target priming and cue-initiated search operate directly on this network. The predicted probability of recalling a primed target from the semantic network, given its test cue, is

$P\left(T^{\prime} / Q\right)=\frac{S\left(Q, T^{\prime}\right)}{S\left(Q, T^{\prime}\right)+S(Q, D)}$,

where total cue-target strength $S\left(Q, T^{\prime}\right)$ is

$S\left(Q, T^{\prime}\right)=S_{q t}+S_{t q}+S_{t^{\prime}}$

cue-distractor strength $S(Q, D)$ is

$S(Q, D)=\frac{\sum_{i=1}^{d} D_{i}}{m}$

where $d=$ distractors and $m=$ number of cue associates, and target-priming probability $P\left(T^{\prime}\right)$ for associates $A_{i j}$ is

$S_{t^{\prime}}=\frac{\sum_{i=1}^{n} \sum_{j=1}^{n} A_{i j}}{n(n-1)}$,

where $n=$ number of target associates.

\section{Results}

To test the predictions of Eq. 1, we used the extralist-cuing database for the cue-target pairs learned under standard conditions (i.e., 3-s study, "remember" instructions). The probabilities of observed and predicted recall were normally distributed around means of $.518(S D=.26)$ and $.392(S D=.16)$, respectively. We computed the error distribution by subtracting predicted from observed recall and plotting the results, and this distribution was normal and shifted to the right because the equation underestimates recall.

The first row of Table 5 presents the results of a multiple regression analysis with Eq. 1 as the sole predictor, and it is well correlated with probability of recall, $r=.567$. Figure 3 shows observed and predicted recall as a function of four categories according to Eq. 1, and as can be seen, it underestimates the observed recall by about the same amount in each category. The transformation to proportions solves the overprediction problem associated with Pier2. Equation 1 uses fewer variables, predicts as well as Pier2, and explains $32.1 \%$ of the total variance in extralist cuing. When the correlation is corrected for the reliability of the free-association and extralist-cuing tasks (Myers \& Well, 1995, pp. 394-395, 472-473), the correlation between predicted and observed recall rises from $r=.567$ to $r=.703$, and the explained variance rises to $49.5 \%$ (see Nelson \& Zhang, 2000, p. 610). Thus, nearly half of the total variance of extralist recall is explained by free-association measures based on links among the words comprising the semantic network. The network can contribute substantially to episodic recall.

\section{Effects of test delay}

Fifteen experiments showed that recall in the extralist-cuing task is negatively affected by test delays filled with solving multiplication problems (e.g., Nelson, Bajo, \& Casanueva,

Table 5 Results of regression analyses for Eq. 1 as the sole predictor, in the first row, and regression comparisons between Eq. 1 against test delay, presentation rate, and study instructions in the successive rows

\begin{tabular}{|c|c|c|c|c|c|c|c|}
\hline & $F$ & $M S_{\text {res }}$ & $R$ & Adj $R^{2}$ & Std. Coeff. & $S E$ & $t$ Value \\
\hline Regression & $F(1,2801)=1,326.00$ & .045 & .567 & .321 & & & \\
\hline Eq. 1 & & & & & .567 & .025 & 36.41 \\
\hline Regression & $F(2,2552)=285.07$ & .048 & .427 & .182 & & & \\
\hline Eq. 1 & & & & & .384 & .026 & 21.43 \\
\hline Test delay & & & & & -.164 & .000 & -9.15 \\
\hline Regression & $F(2,3220)=778.08$ & .045 & .571 & .325 & & & \\
\hline Eq. 1 & & & & & .565 & .023 & 39.02 \\
\hline Rate & & & & & .084 & .010 & 5.81 \\
\hline Regression & $F(2,369)=930.25$ & .045 & .597 & .356 & & & \\
\hline Eq. 1 & & & & & .551 & .022 & 39.78 \\
\hline Instructions & & & & & .207 & .009 & 14.93 \\
\hline
\end{tabular}




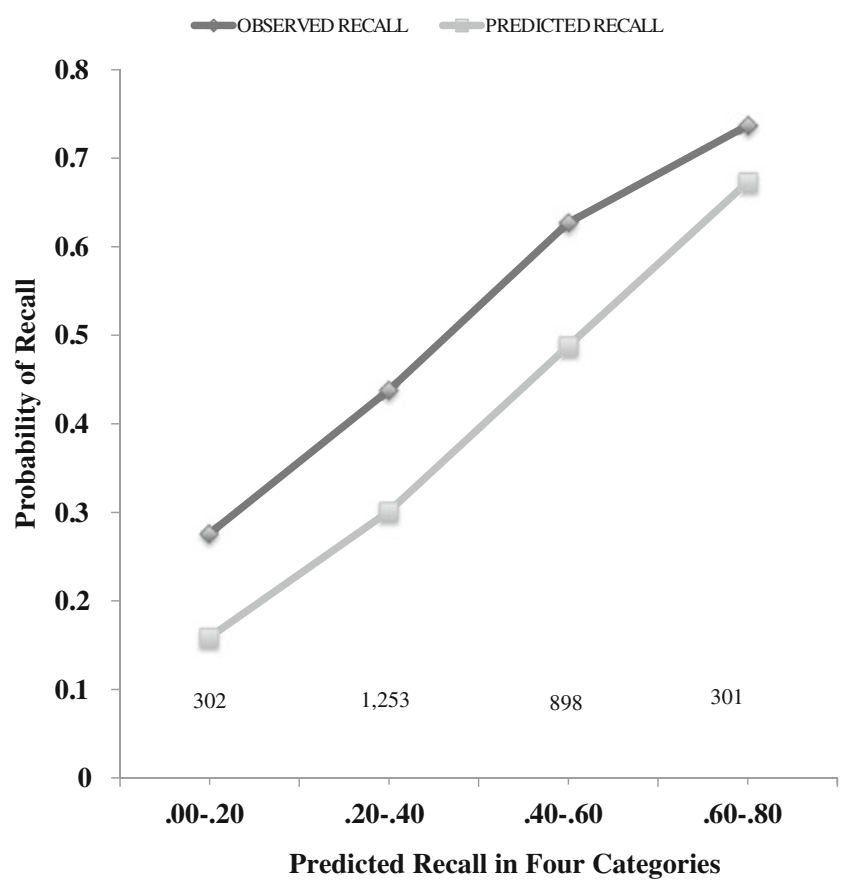

Fig. 3 Observed and predicted probabilities of recall as a function of the Eq. 1 predictions, in four categories

1985; Nelson et al. 2007; Nelson et al., 1998). Their data were rescored at the item level and pooled over experiments in order to portray the effects of delays at 2, 7, 10,12, 22, and $1,442 \mathrm{~min}$. Two minutes were added to each delay in order to accommodate study-to-test time that would include time for reading the test instructions. A multiple regression analysis that included the four variables plus test delay as predictors was significant $\left[F(5,2549)=138.82, M S_{\text {res }}=.046, R=.46\right.$, $\left.R^{2}=21.2 \%\right]$. The coefficients or weights allocated to cuetarget strength $S_{q t}$, target-cue strength $S_{t q}$, cue-distractor strength $S_{q d}$, neighborhood density $S_{t}$, and delay by the regression equation were all significant, and the respective correlations between each source and observed recall were $.32, .14,-.21, .22$, and -.19 . When the interactions between each source and delay were included as predictors, none were significant. The absence of delay interactions is inconsistent with experimental findings showing that the strong-weak difference for each variable declines as delay increases (see also Sahakyan \& Goodmon, 2010). Examinations of mean recall with the data split along strong-weak levels for each variable indicated that this trend was apparent in the database, but the effects, though smaller, were apparent even after $24 \mathrm{~h}$. Finally, as is shown in the second row of Table 5, Eq. 1 and delay were significant predictors in a simultaneous multiple regression, and each predictor was significantly correlated with recall, $r \mathrm{~s}=.40$ and -.19 .

A $\log -\log$ plot of probability of recall and test delay approximated a straight line, and a power function fitted to recall as a function of untransformed values explained $88 \%$ of the variance that could be explained, with an intercept constant of .541 and a slope of -.109. Effects of delay were incorporated into Eq. 1 by weighting it by $t^{-\alpha c}$, where $t$ is time in minutes, $\alpha$ is decay rate estimated by the power function, and $c$ is a context parameter $(c \geq 1)$ that is estimated from the data and accounts for differences in how the retention interval is filled (e.g., with multiplication problems, studying additional lists, or room and experimenter changes). When the effects of test delay are taken into account, Eq. 1 becomes

$P\left(T^{\prime} / Q\right)=\left[\frac{S\left(Q, T^{\prime}\right)}{S\left(Q, T^{\prime}\right)+S(Q, D)}\right]\left(t^{-\propto c}\right)$.

Figure 4 shows probability of recall as a function of test delay using Eq. 5 with $c=1$. Recall declines sharply over the first $20 \mathrm{~min}$ and levels out after $24 \mathrm{~h}$. When Eq. 5 was used as a single predictor of recall in the multiplication problem database, it emerged as a significant predictor, $F(1,2553)=686.5, M S_{\text {res }}=.046, R=.46, R^{2}=21.2 \%$.

We attribute the effects of test delay to decay and the loss of context information (e.g., Nelson \& Goodmon, 2003; Nelson, Goodmon, \& Akirmak, 2007; Nelson et al. 2007; Sahakyan \& Goodmon, 2010). Theoretically, the target is primed and encoded in conjunction with environmental context cues sampled during study, and asking subjects to switch attention from the study list to math problems and the like disrupts the recovery of these cues. Additional support for the importance of context has come from studies showing that changes in the physical environment - produced by changing rooms, experimenters, or both between study and test - as well as requiring the study of additional lists during the delay, reduce the probability of recall. However, neighborhood density effects are attributed to target priming. Forgetting describes why recall declines, but it does not explain why priming effects are reduced but still apparent after $24 \mathrm{~h}$, when activation normally decays within milliseconds in speeded lexical and semantic decision tasks. In contrast to the case in speeded tasks, density-induced target-priming effects somehow persist in extralist cuing, and we suggest a reason below.

\section{Effects of study condition}

Study conditions varied by means of presentation rate and instructions have robust effects on recall in extralist cuing. The database included 1,265 pairs encoded under nonstandard conditions. In some experiments, presentation rate was varied under the otherwise standard conditions, and recall increased with slower rates, averaging .47 ( $S E=.02, n=210$ pairs), .52 $(S E=.01, n=2,803)$, and $.60(S E=.02, n=210)$ for rates of 1.5, 3.0, and $6.0 \mathrm{~s}$ (see, e.g., Nelson, Schreiber, \& McEvoy, 1992). With Eq. 1 and rate as predictors, the results in the third row of Table 5 indicate that the regression and both predictors are significant. The correlations with recall for Eq. 1 and rate 
Fig. 4 Observed and predicted probabilities of recall as a function of minutes of test delay
Probability of Recall

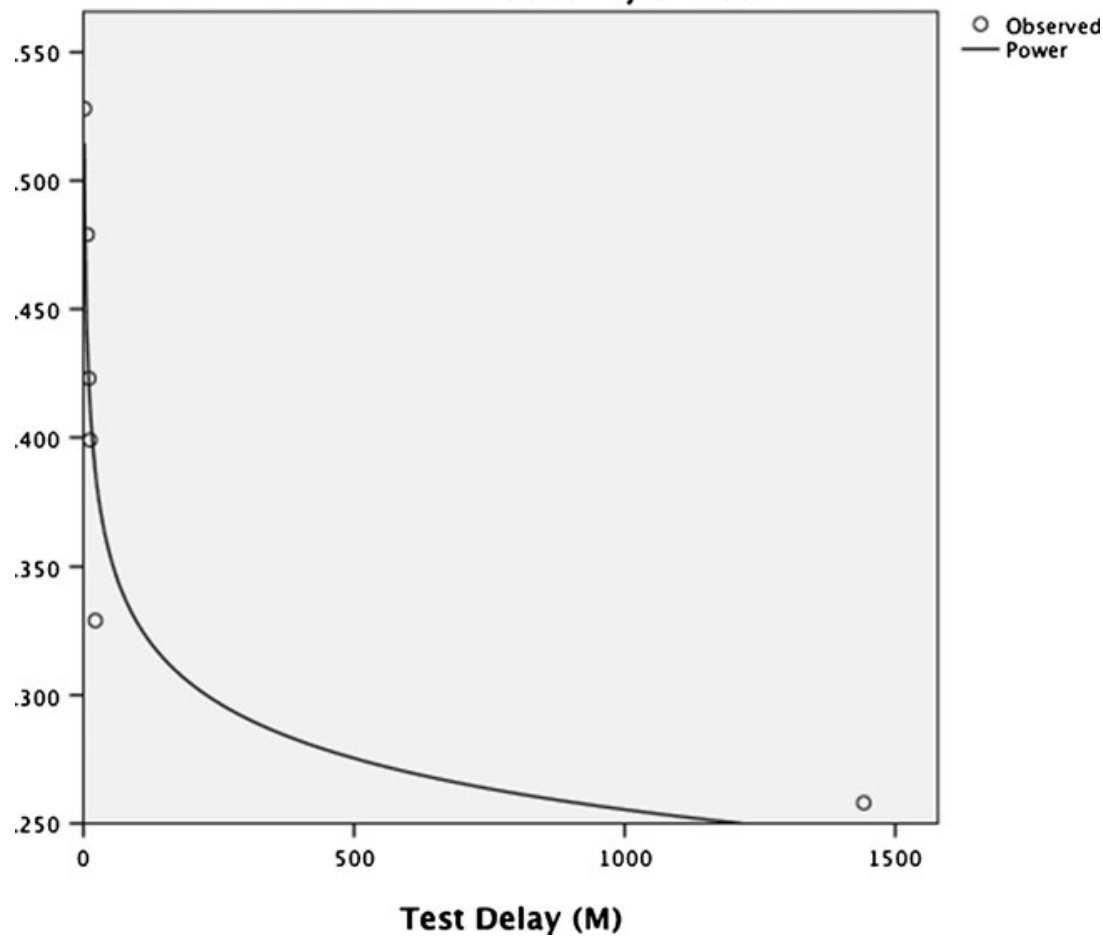

are $r=.57$ and .08 , and they explain $98.1 \%$ and $1.9 \%$ of the variance that can be explained. In extralist cuing, the contribution of semantic memory is substantially greater than the contribution of additional study time.

In other experiments, different groups of subjects have processed the targets by naming their vowels, trying to remember them in the standard condition, or rating their concreteness (e.g., Nelson et al. 2007). Recall increased with elaboration and averaged $.39(S E=.02, n=210), .52$ $(S E=.01, n=2,803)$, and $.68(S E=.02, n=427)$ for naming vowels, "remember" instructions, and rating concreteness. We coded instructions as $-1,0$, and +1 and entered it along with Eq. 1 as a predictor in a multiple regression. Table 5 shows that the regression equation was significant and that Eq. 1 and the study instructions contributed significantly to recall. Equation 1 and instructions were correlated with recall at $r=.56$ and .23 , and an unforced stepwise regression indicated that they explained $87.9 \%$ and $12.1 \%$ of the variance that could be explained.

Consistent with study time, preexisting information in the semantic network contributes more to recall in extralist cuing than does level of processing. Recall in extralist cuing relies on both semantic and episodic manipulations, but it depends more on the contribution from the semantic network than on explicitly defined encoding procedures. The network captured in Eq. 1 explains more variance than other predictors because the test cue is absent during study and target recall hinges on using the test cue to access the target within the semantic network. In experiments, these two sources of information generally do not interact, but when they do, the interaction is weak and potentially the result of ceiling effects. These results suggest that semantic network variables and episodic encoding operations $(E)$ have additive effects on recall in the extralist task, and that total cuetarget strength (Eq. 2) can be re-represented as

$S\left(Q, T^{\prime}\right)=S_{q t}+S_{t q}+S_{t^{\prime}}+E$.

Developing an equation for $E$ is beyond the scope of this article, but we will consider two implications of Eq. 6. The first is that encoding operations applied according to the study instructions add their effects directly to the total cuetarget strength $S\left(Q, T^{\prime}\right)$. Total strength is greater when the study instructions focus on semantic processing or remembering rather than on naming vowels. The second implication is that two different types of information contribute to target recall in the extralist-cuing task, one based on episodic encoding operations and one based on implicitly activated semantic information. The episodic processes are tied to how targets are encoded, to study time, and to general context cues, and hence to recollective retrieval processes. The implicit processes are linked to the strength of the cuetarget relationship and to target priming, and hence to automatic, unaware retrieval processes.

Pier incorporates both dual encoding and dual retrieval processes in explaining extralist cuing. Pier3 assumes that the appearance of a target can prime its representation in the semantic network in accordance with its neighborhood 
Fig. 5 The representation of Atom in semantic memory can be strengthened by priming effects produced by its neighborhood density in the network and by encoding processes in working memory. Although priming effects are generally short-lived, they can be long-lasting when a primed version of a target word is encoded in working memory

\section{Semantic Memory}

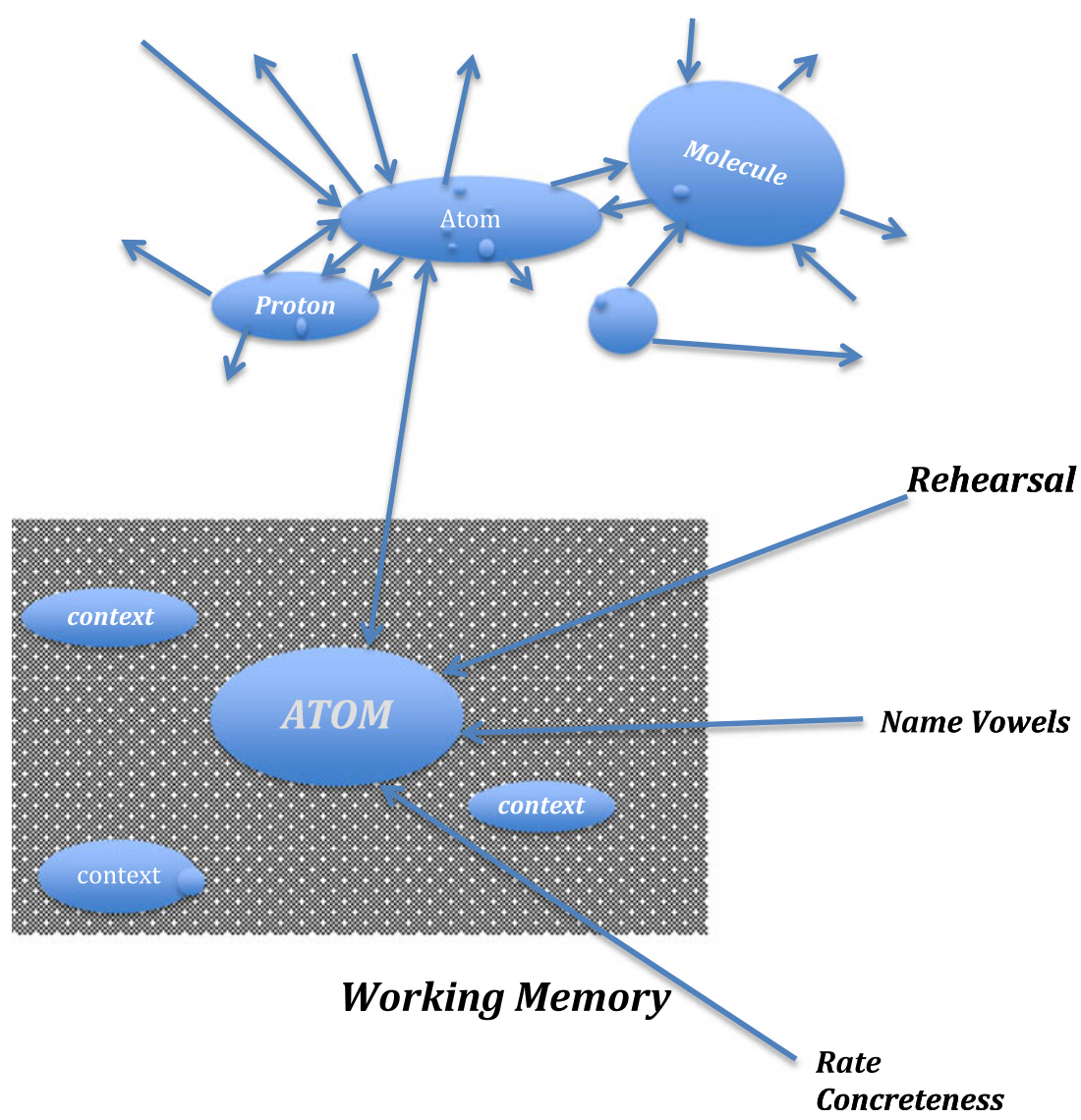

density, and that regardless of its primed state, a target is automatically loaded into working memory and subjected to encoding operations controlled by the study instructions. Both target-priming effects and the encoding processes applied to the target in working memory strengthen its representation in the semantic network. As is illustrated in Fig. 5, the studied word Atom is represented in the network, and its appearance in the experimental setting activates this representation, with neighborhood density theoretically affecting its primed strength. The application of working memory operations to a primed representation of Atom adds to its strength in the semantic network, so that any cue linked to it in the network has an increased likelihood of selecting it as a response during testing. Importantly, we assume that Atom's associates serve only to prime its representation in semantic memory, and that they are not processed in working memory. We found small implicit associative strengthening effects in an early experiment (Nelson, Bajo, McEvoy, \& Schreiber, 1989), but several unpublished experiments by Schreiber failed to replicate such effects.

As is suggested in Fig. 5, primed targets such as Atom are encoded in the presence of elements of the environmental context that are simultaneously represented in working memory, so context becomes associated with the primed target (cf., Raaijmakers \& Shiffrin, 1981). Atom's representation in the network is temporarily strengthened by all sources of information, including priming, explicit encoding operations, and context. When Proton is presented as a reminding cue, it initiates a search via its associative array. Atom is now more likely to be selected than are Proton's other associates, if it has been primed and explicitly encoded via study instructions, and this encoding occurs in the presence of information about the context of the experience. Relative to Proton's distractors, Atom now has a greater chance of being recalled, because its state in the semantic network has been strengthened via both episodic and implicit processes, and this state explains why target-priming effects can be long-lasting in extralist cuing: They last when target words are encoded in a primed state in working memory.

Primed free association

Primed free-association and extralist-cuing tasks are similar because the same list of targets and test cues can be used along with the same study conditions, and the hypothesis that primed information is retrieved from semantic memory 
predicts that the same variables will be important in both tasks. The main difference between these tasks occurs during testing. In primed free association, subjects are asked to free-associate the first related word to come to mind for each test cue, under a cover story suggesting that norms are being collected for another experiment, and they are not told that the test cues are related to the targets. To reduce the probability of their discovering the relationship, the test often begins with unrelated "cues" and then continues with a random mix of equal numbers of related and unrelated cues (e.g., Chappell \& Humphreys, 1994; Humphreys, Tehan, O'Shea, \& Bolland, 2000; Nelson \& Goodmon, 2002; Zeelenberg, Shiffrin, \& Raaijmakers, 1999). We focus on the probability of recalling the target as a function of the variables that comprise Eq. 1, to determine whether they have similar effects in extralist cuing and primed free association. The intentional-recall instructions used in extralist cuing make subjects aware of the relevance of the target words just seen in the study context and the cue-target relationship, whereas free-association instructions relax awareness on both counts. Neither the study context nor the semantic relationships between the cues and targets are ever mentioned in the test instructions. Relaxing awareness decreases recall, but the variables that affect intentional recall should have similar effects in primed free association if both tasks are relying on the same semantic network.

\section{Results}

Previous experiments confirmed this expectation and showed that target recall was greater when $S_{q t}$ and $S_{t q}$ were stronger, as well as when $S_{t^{\prime}}$ was higher. As in the extralist-cuing task, they also showed that target recovery is more likely when attention is directly focused on target meaning during study as compared to standard "remember" instructions, and when testing is immediate as opposed to delayed (Goodmon \& Nelson, 2004; Nelson \& Goodmon, 2002; Nelson et al. 2007).

The data from these and related experiments involving immediate tests were rescored at the item level and used to create a primed free-association database that included 470 cue-target pairs, probability of target recall, and the predictor measures. Although recall is very low in primed free association, the results of a simultaneous multiple regression analysis, shown in Table 3, indicated that the regression was significant, as were the effects of each of the four predictors. Table 6 presents the correlations for each variable, and note that although $S_{q d}$ was not varied in any experiments, it nonetheless emerged as a significant negatively correlated predictor. As in extralist cuing, target recall increased significantly as a positive function of $S_{q t}, S_{t q}$, and $S_{t}$, and it declined as cue-todistractor strength $S_{q d}$ increased. An unforced stepwise regression showed that recall was significantly affected by all four network variables, with $S_{q t}, S_{t q}, S_{q d}$, and $S_{t^{\prime}}$ explaining $23.9 \%$, $4.0 \%, 3.2 \%$, and $0.4 \%$ of the total variance. The effects of all four variables were reduced in primed free association as compared to extralist cuing, but each variable was significant, and the effects of $S_{t}$, although quite small, indicate that target priming still affected recall.

\section{Predicting priming}

A multiple regression analysis with probability of target recall as the dependent measure and Eq. 1 as the predictor was significant, but it overpredicted recall, $F(1,468)=118.36$, $M S_{\text {res }}=.023, R=.45, R^{2}=20 \%$; the mean predicted and observed recall were .33 and .19 . Observed recall is very low in primed free association when directly compared to extralist cuing, where recall can be twice as high (Nelson et al. 2007). This low recall arises because subjects are unaware of the importance of the study episode and the cue-target relationships. Weighting Eq. 1 by .5 brings the predicted mean target recall more in line with the observed recall, and although it must be adjusted, Eq. 1 explains $20 \%$ of the total variance and is an acceptable predictor of primed free association. Target recall is thus based on the semantic network, even in the absence of an intent to recall.

\section{Studying semantically related pairs}

In extralist cuing, each target is presented in the absence of semantic context during study, but in this section we will consider a task in which the target is studied in the context of a semantically related word. A word seen in either context implicitly activates its closest associates (e.g., in lexical

Table 6 Correlations (above diagonal) and partial correlations (below) for primed free association

\begin{tabular}{llccccc}
\hline & $S_{q t}$ & $S_{t q}$ & $S_{q d}$ & $S_{t^{\prime}}$ & Study Condition & $r$ With Prob. of Recovery \\
\hline$S_{q t}$ & - & -.10 & -.37 & .02 & -.13 & .49 \\
$S_{t q}$ & -.12 & - & .12 & .07 & .12 & .11 \\
$S_{q d}$ & -.21 & .15 & - & -.31 & .17 & -.37 \\
$S_{t^{\prime}}$ & -.14 & .10 & -.29 & - & -.11 & .16 \\
Study Condition & -.14 & .07 & .13 & -.09 & - & .06 \\
Partial $r$ With Prob. of Recovery & .45 & .19 & -.23 & .09 & .17 & - \\
\hline
\end{tabular}


decisions, both meanings of a polysemous word are momentarily activated; Kintsch, 1988). However, the effects of this broad activation on recall could not be more different in the two contexts. When seen in isolation in the extralist task, target meaning is uncertain, and the activation of related words provides an internally generated semantic context that primes the target's representation to varying degrees. Theoretically, this primed representation is loaded into and studied in working memory, thus preventing the rapid decay of target priming. For example, when Bolt is studied in isolation, both its "fastener" and "storm" meanings support and prime its activation state, and because the target is rehearsed and encoded in this state, both meanings affect recall, even when Bolt is cued by Screw or Lightning, which specify one meaning or the other (Gee, 1997). Both meanings contribute to Bolt's priming level during study, so they affect recall even when search is initiated by only one of its meanings.

In contrast to semantic isolation, encountering a pair of semantically related words during study tends to produce a semantically specific encoding (Tulving \& Thomson, 1973). For example, in the intralist-cuing task, after studying Screw-Bolt, Screw is an effective cue, whereas Lightning fails dramatically (Nelson et al. 1979). Following Kintsch (1988), we assumed that both the context cue Screw and its target Bolt activate their associates during study, and that this broad activation collapses to a specific meaning provided by the semantic context implied by the word pairing. Because the target's meaning is specified during study in intralist cuing, its associates are no longer needed to support its semantic state, and as a consequence, the density of the target's semantic neighborhood has no effect. The cue-target pairing can be directly loaded into working memory, where it can be rehearsed and encoded. Furthermore, in intralist cuing, collapse of activation is observed regardless of whether or not a target word has multiple, distinct meanings, because its meaning is more certain than when the word is semantically isolated (Nelson et al., 1979).

Three findings reinforce the collapse assumption. First, in intralist cuing, the target is studied in the presence of a semantically related context word that is used as the test cue, and effects related to its associates are mostly eliminated (Holley \& McEvoy, 1996; Nelson, Gee, \& Schreiber, (1992), Nelson, Schreiber, \& McEvoy, (1992)). This collapse occurs when the pairing is studied and is not produced by the test cue itself, because collapse is observed even when recall is prompted by a rhyme cue linked to the target's name (Nelson et al. 1992). Second, the presence and timing of the semantic context word in relation to its target is critical (Nelson et al. 1992). When the context word appears slightly before or after the target, effects related to the target's associates are apparent once again, but the extent of these effects falls between the levels during the context-absent and the context-simultaneous conditions. Short delays in the appearance of the context word introduce semantic uncertainty and reveal the effects of the target's associates. Total collapse of the target's associates depends on simultaneously experiencing the cue-target pair. Finally, as compared to when unrelated facts are memorized, facts organized around a semantic theme eliminate fan effects (Anderson, 1983; Reder \& Anderson, 1980) - that is, increases in latencies with the number of a word's associates. Fan effects are distractor effects, and as with word pairs, encoding facts in a specific semantic context sharply reduces distractor effects.

On the basis of these findings, we suspected that of the four predictors in Eq. 1, only cue-to-target strength $S_{q t}$ should affect intralist cued recall, because the test cue must be used to recover the cue-target pair in the semantic network. In order to evaluate the effects of the four components of Eq. 1 on a larger scale, we rescored the data from 12 intralist-cuing experiments and created a database of 802 pairs. In the first analysis, the four variables were used as predictors and the data were restricted to pairs presented at a 3 -s rate and studied under instructions to remember the pair and that the word appearing on the left would be the test cue. The regression was significant, as is shown in Table 3, and cue-to-target strength $S_{q t}$ is the only significant predictor. In a second analysis, we included cue-to-target strength $S_{q t}$, presentation rate, and target frequency as predictors. The regression was significant $\left[F(3,798)=40.78, M S_{\text {res }}=.035\right.$, $R=.37, R^{2}=13.0 \%$ ], as were all three predictors. For $S_{q t}$, presentation rate, and frequency, the coefficients were .31 $(t=9.24), .18(t=5.30)$, and $-.14(t=-4.15)$, and they explained $8.4 \%, 2.8 \%$, and $1.8 \%$ of the total variance. The recall means at study times of $1.5,3.0,5.0$, and $6.0 \mathrm{~s}$ were $.75(S E=.02, n=93), .74(S E=.01, n=590), .81(S E=.02$, $n=47)$, and $.87(S E=.02, n=93)$.

As compared to extralist cuing, in intralist cuing, cue-totarget strength and study time had much reduced effects, and target-to-cue and cue-to-distractor strengths and, importantly, the target's neighborhood density had no observable effects. These contrasts indicate that the semantic network responds dynamically to the context provided by the task at hand. In extralist cuing, a word's meaning as encoded during study tends to be distributed across its semantic contexts, whereas in the intralist task, it tends to be confined and specific, which comes as no surprise (e.g., Tulving \& Thomson, 1973). What is most interesting is the extralistcuing result showing that the semantic network supplies meaning when a word is experienced out of context and delivers what it has activated to a working memory system that encodes this information for future use. The semantic network responds dynamically to context and to memory systems that have evolved for encoding recent events. Next, we will consider effects of neighborhood density in singleitem recognition. 


\section{Recognition}

In single-item recognition, subjects study a long list of words, followed by a second list that is usually twice as long and includes equal numbers of studied and new words. During study, subjects process the words in some way, and the second list tests recognition by having the subjects separate the test words into two categories, "old" and "new." In this task, the test cue is the target itself, so of the four variables used in cuedrecall studies, only neighborhood density is relevant. Nelson et al. (2001) asked subjects to study words that varied in density and printed word frequency (see also Fisher \& Nelson, 2006; Nelson et al., 1998). Theoretically, targets with greater density would be more likely to be correctly recognized when "old" and correctly rejected when "new." This finding holds across variations in frequency and differences in study instructions ("rate concreteness," "name vowels," or "remember").

To deepen our understanding of these results and to control for the effects of correlations between the predictor variables, we set up a recognition database that included the targets from all of our experiments. Targets were represented in one column, with four measures of recognition performance ( $d^{\prime}, A^{\prime}$, hits, and false alarms) and the predictor variables represented in the remaining columns. Table 3 shows the results of a simultaneous multiple regression of the pooled data and indicates that all of the manipulated variables significantly predicted $d^{\prime}$ (and the other measures). Printed frequency effects are not shown in the table, but as expected they had a very strong effect, with a coefficient of $-.36, S E=.031$, $t=-17.13$. The study instructions (rate concreteness, name vowels, or remember), frequency, and neighborhood density were significantly correlated with $d^{\prime}$ at $r=.38,-.37$, and .12 , and these correlations explained $50.6 \%, 46.9 \%$, and $1.7 \%$ of the variance that could be explained. Although the effect was small, higher levels of neighborhood density were positively correlated with single-item recognition.

In the recognition task, word frequency explains more variance than does neighborhood density. A similar analysis on extralist cuing disclosed a reversed pattern. Of the explained variance, frequency and target density explained $2.7 \%$ and $49.1 \%$, respectively. In both tasks, study instructions explained about the same amount of variance, but recognition was more affected by distinctive letter patterns (e.g., Malmberg, Steyvers, Stephens, \& Shiffrin, 2002; Shiffrin \& Steyvers, 1997), whereas extralist cuing was more affected by neighborhood density and target-priming effects. Both tasks rely on both nonsemantic and semantic information, but the same information plays different roles in the two tasks. In recognition, the target is used as a cue for itself, and its orthographic features play a more important role than its semantic neighborhood. Alternatively, extralist cuing relies on an external cue, and thus is forced to rely on the semantic network. Recognition appears to depend more on matching the target's name, whereas extralist cuing depends more on searching the network (e.g., Humphreys, Bain, \& Pike, 1989).

\section{Summary of neighborhood density effects}

The experimental and correlational findings indicate that neighborhood density effects linked to the target facilitate target recall and recognition to varying degrees. As density increases, words are more likely to be recognized as studied and to be rejected when not studied, and they are more likely to be recalled when prompted by extralist test cues. In all of these tasks, a target is experienced in semantic isolation during study, and semantic memory responds by activating its semantic neighborhood. Observing density effects appears to be contingent on both the absence of semantic context during study and the presence of a related extralist cue or a self-cue that provides access to its primed and encoded representation in semantic memory.

The findings also show that the magnitudes of neighborhood density effects in extralist cuing do not interact with study time or level-of-processing manipulations. Density effects are manifested at short and long study times, and regardless of whether subjects are naming vowels, rehearsing, or rating semantic attributes. Though the effects are small, density also influences target recovery in primed free association, where the importance of the study context and the cuetarget relationship are never mentioned. Finally, although target recall declines quickly as a function of test delays, target density effects are reduced but still apparent after $24 \mathrm{~h}$.

However, target density effects are not evident when targets are presented simultaneously with a semantically related word that specifies their meanings, and though this can be interpreted as indicating that the neighbors are not activated, the speeded decision literature suggests otherwise (e.g., Kintsch, 1988): The target's associates appear to be activated, but this activation rapidly collapses because the associates are irrelevant when target meaning is specified by the context. The processes underlying neighborhood density effects, as well as their collapse, appear to be automatic, unconscious, and fast. Activation affords rapid access to prior knowledge in the semantic network and is most relevant when the prevailing semantic context is nonexistent or ambiguous. In order to comprehend what it is experiencing, the brain rapidly provides potentially relevant semantic contexts. Although activation is normally treated in the speeded decision literature as an ephemeral event lasting milliseconds, we suggest that neighborhood density effects survive in semantically uncertain contexts because primed targets are encoded in working memory. A target's primed representation functions as a foundation for encoding processes performed upon it, and such encoding ensures longer-lasting priming effects. The question of why the semantic system operates in this way seems 
straightforward: Given semantic uncertainty, activating prior knowledge allows the system to quickly grasp the meaning of current events in the broadest possible sense, in order to anticipate unknown specific responses to future events. When a future event arrives in the form of a retrieval cue, a primed and encoded target is more likely to be selected within the test cue's noisy set of related distractors.

\section{Sources of neighborhood density effects and target priming}

The most important question about the effects of neighborhood density has been postponed, and it concerns the underlying process: When the target is studied in semantic isolation, how does the relative density of the links comprising its neighborhood affect its recall and recognition? We assume that neighborhood density primes the representation of the target to greater or lesser degrees, but how does the activation of the target's associates prime the target? In this section, we offer two explanations: spreading activation and quantum-like entanglement.

\section{Spreading activation}

Initially, we turned to the Collins and Loftus (1975) spreadingactivation explanation. When applied to target-priming effects, spreading activation implies that seeing the target spreads activation to and among its associates, and then back to the target through associate-to-target links (Nelson et al., 1998). Spreading activation is inherently a sequential process, whereby activation moves from one word to the next along the links in the network. To illustrate this idea, Fig. 6 represents target $T$ with links to two associates, one associate-to-associate link, and one associate-to-target link. In the two-step loop, activation spreads from $T$ to Associate 2 and back to $T$. In the three-step loop, it travels to Associate 1, to Associate 2, and then to $T$. According to this hypothesis, the effect of the link from Associate 1 to Associate 2-or, more generally, the

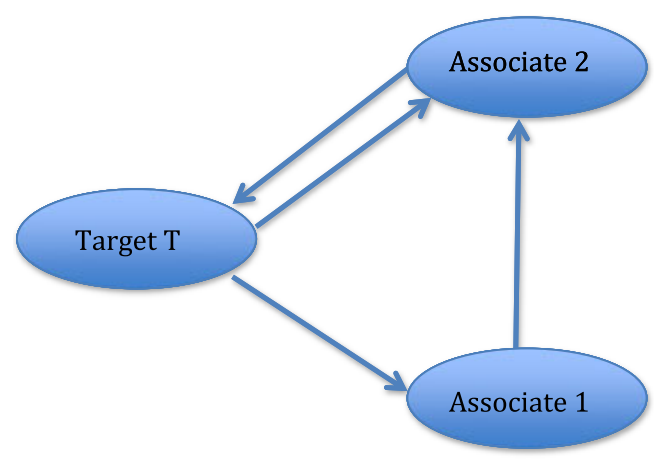

Fig. 6 Target $T$ activates target-to-associate, associate-to-associate, and associate-to-target links effects of associate-to-associate links - arises because they direct activation back to the target, rather than to words outside its neighborhood.

According to spreading activation, target-priming effects are contingent on the probability of links back to the target, and this contingency predicts that effects of associate-to-associate links must be greater when more associate-to-target pathways exist. While this account is reasonable, the results of several experiments that manipulated the proportions of both types of links in a factorial design rejected this alternative (Nelson, McEvoy, \& Pointer, 2003). They showed that associate-to-associate effects are robust as compared to associate-to-target effects, and more importantly, that the expected interaction between the two variables does not emerge; the target-priming effects of associate-to-associate links are just as apparent when there are few associate-to-target links as when there are many. In addition, the preliminary regression analysis of extralist cuing showed that associate-to-target links are not significantly correlated with recall when other variables are controlled (Table 2). Moreover, Bruza, Kitto, Nelson, and McEvoy (2009) pooled the data from the experiments above and found that a spreading-activation equation was a poor predictor of recall $(r=.35)$ as compared to Pier2 $(r=.57)$. Hence, experiencing a word does not appear to activate its nearest neighbors sequentially. We assume that neighbors are activated in parallel, but this assumption begs the question of how such an event can be modeled, and we turned to the concept of quantum entanglement for an alternative explanation.

Semantic entanglement

The mathematics of quantum mechanics is being used to generate a variety of models in the social sciences, and the purpose of this section is to show how it can be used to model target priming in extralist cuing (Aerts, 2009; Busemeyer \& Bruza, 2012; Busemeyer, Pothos, Franco, \& Trueblood, 2011; Gabora \& Aerts, 2002; Pothos \& Busemeyer, 2009). Entanglement is a fundamental concept in quantum mechanics that arises when two physical entities (such as electrons and photons) are subjected to "preparations," or what cognitivists might call "procedures," and these preparations produce highly correlated experimental findings. Once the entities are entangled, measuring the state of one of them determines its state and simultaneously provides information about the state of the other entity, even when it is so distant that communication between them is impossible (see Bruza et al., 2009, for more detail on how quantum theory is applied to primed target strength; Greenstein \& Zajonc, 2006, for an introduction to experimental work; Laloe, 2001, for a review of quantum theory; Maudlin, 1994, for a discussion of entanglement and its implications; and Turvey \& Moreno, 2006, for a rationale behind using physical metaphors to understand the semantic network). 
Once entangled, the entities can be represented as a "superposition state," illustrated in the following equation that describes a situation that arises before the spin states of two particles are measured. Particle 1 has a potential of spinning "down" while Particle 2 is spinning "up," or, alternatively, Particle 1 has a potential of spinning "up" while Particle 2 is spinning "down":

$\psi_{\text {before measurement }=\frac{1}{\sqrt{2}}\left[\left(\downarrow_{1} \uparrow_{2}\right)+\left(\uparrow_{1} \downarrow_{2}\right)\right]} \cdot$

This superposition represents an indefinite state, and the actual spin states of two entangled electrons cannot be known until spin direction is measured. Measuring the state of one of the electrons causes it to collapse to spinning "up" or "down." For example, if Particle 1 is measured, the state could collapse to one state or the other:

$\psi_{\text {after measurement }}=\frac{1}{\sqrt{2}}\left[\left(\downarrow_{1} \uparrow_{2}\right)\right]$

or

$\psi_{\text {after measurement }}=\frac{1}{\sqrt{2}}\left[\left(\uparrow_{1} \downarrow_{2}\right)\right]$

The important point is that measuring the state of Particle 1 predicts the state of Particle 2. In the "after" measurement state, if Particle 1 is observed as spinning down, we know that Particle 2 is spinning up, regardless of its distance from Particle 1. Alternatively, if Particle 1 is observed as spinning up, we know that Particle 2 will be observed as spinning down. Once entangled, measuring the state of one particle determines its state and, simultaneously, predicts the state of the other particle.

To say the least, words differ from physical entities, but certain abstract similarities make the comparisons worth considering. For example, in both scientific disciplines entities become entangled in some context, and measuring the state of one of them simultaneously provides information about its state and the states of correlated entities. In our case, observing target-priming effects reveals the activation states of a target's associates. Our findings show that links among the target's associates prime its representation in accordance with its neighborhood density. They also suggest that neighborhood density effects are contingent on parallel activation. An entangled state has to be "prepared," and this state emerges when the target's neighborhood in the semantic network is simultaneously activated. Theoretically, simultaneous activation generates an indefinite superposition state in the experimental context, and quantum-like entanglement provides a plausible hypothesis for explaining how neighborhood density effects emerge from links among its associates (Bruza et al., 2009; Busemeyer \& Bruza, 2012; Galea, Bruza, Kitto, Nelson, \& McEvoy, 2011).
The preparation phase occurs during study, when the target appears on the screen. Its appearance puts the target's network representation into a superposition state with its associates. The model assumes that each of the associates is activated with a probability of 0.0 and that each of the associates is activated with a probability of 1.0. A word's representation is recovered from the network in an indefinite state in which each of its associates is not and is activated. This state collapses to one activation state or the other when it is observed in context, and theoretically, this occurs when the representation appears in working memory, where it is observed, read aloud, and rehearsed, or rated on some attribute. The superposition collapses to a definite state of being either not primed or primed when the observer becomes aware of the target's existence in the experimental context. Becoming aware of a word in the setting is analogous to "measuring" the state of an electron, in the sense that this measurement produces a definite state.

\section{Entanglement model details}

We will first illustrate how target priming is computed in the entanglement model, and then describe the underlying conceptualization. Table 7 presents a matrix representation of target $T$ and its three associates. It shows how the probability of activating each associate is calculated in the bottom row, and how these associates affect target priming. Theoretically, each probability measures the degree to which an associate is activated by links from $T$ 's other associates, and Eq. 14 below shows how the entanglement model combines the probabilities and predicts target priming. Note that the equation indicates that each associate contributes to priming, regardless of its semantic distance from the target (readers may wish to skip ahead to superposition Eq. 11 after examining this table).

The model treats the associates as "qubits" in a manybodied system. A qubit is a "quantum bit" and is a quantum analogue of a classical bit. In classical probability theory, a bit takes on a definite value of 0 or 1 , whereas a qubit exists as a weighted sum of both of these states that defines the superposition. A classical bit represents information in a binary fashion on the basis of logic states of "off" or "on." In contrast, a qubit can represent multiple states simultaneously for brief periods, because both alternative outcomes are concurrently represented, along with a weighting term that relates to their probability of occurring.

In quantum theory, "off" and "on" are represented in kets $|0\rangle$ and $|1\rangle$, which are called basis states that represent the context of a quantum measurement. ${ }^{2}$ Hence, the superposition state of a system $\psi$ is written

\footnotetext{
$\overline{{ }^{2} \text { The symbol }|\rangle}$ is called a "ket." In quantum mathematics, kets provide a context for describing probabilities - for instance, $|0\rangle$ and $|1\rangle$ are read as "ket 0 " and "ket 1."
} 
Table 7 Matrix representation of Target $T$ with three associates $a$, the links among them (reading along each row), and the probabilities ( $p$ ) that an associate will be activated

\begin{tabular}{llll}
\hline Target & $a_{1}$ & $a_{2}$ & $a_{3}$ \\
\hline$a_{1}$ & - & 1 & 1 \\
$a_{2}$ & 1 & - & 1 \\
$a_{3}$ & 0 & 0 & - \\
Probability & $p_{\mathrm{a} 1}=(1+0) \div 3(3-1)=.167$ & $p_{\mathrm{a} 2}=(1+0) \div 3(3-1)=.167$ & $p_{\mathrm{a} 3}=(1+1) \div 3(3-1)=.3333$ \\
\hline
\end{tabular}

$|\psi\rangle=\alpha|0\rangle+\beta|1\rangle$,

where $\alpha$ and $\beta$ represent "probability amplitudes." When $\alpha$ and $\beta$ are squared, they become probabilities; for instance, $\alpha^{2}$ is the probability that a value of 0 is found, and $\beta^{2}$ is the probability that a value of 1 is found. In quantum probability theory, the indefinite superposition state collapses to one of the specified states when a "measurement" is applied. In our case, the activation state of a target is indefinite until it comes into awareness, which causes its superposition state to collapse to the "not activated" $|0\rangle$ or the "activated" $|1\rangle$ state.

Figure 7 illustrates the system of related associates shown in Table 7 as a set of three qubits. Each qubit provides a spatial representation for one of the target's associates, represented as a vector of length 1.0 for each associate. The probability of a qubit collapsing to one state or the other is related to the squared projection of the qubit state (the vector) down to the basis state defined by the horizontal axis. This projection can be envisioned as drawing a perpendicular line from the tip of the vector onto the basis state. In quantum theory, probabilities are based on the Pythagorean theorem $a^{2}+b^{2}=c^{2}$, so taking the square root of the probability amplitude produces the probability. Thus, the probability of an activated target is equal to the square root of the projection onto the $|1\rangle$ basis state, and the probability of a nonactivated state is equal to the square root of the projection to the $|0\rangle$ basis state. These ket states take the context of activation into account, so that the model is inherently contextual, because the probability of activation changes with changes in the context (Isham, 1995). Making use of this procedure, we can write a superposition state for each of the target's associates $a_{1}, a_{2}$, and $a_{3}$ as

$\left|a_{1}\right\rangle=\sqrt{\bar{p}_{a 1}}|0\rangle+\sqrt{p_{a 1}}|1\rangle$,

$\left|a_{2}\right\rangle=\sqrt{\bar{p}_{a 2}}|0\rangle+\sqrt{p_{a 2}}|1\rangle$,

$\left|a_{3}\right\rangle=\sqrt{\bar{p}_{a 3}}|0\rangle+\sqrt{p_{a 3}}|1\rangle$,

where a bar over $p$ indicates "not activated."

We take all three states in Eqs. 8-10 and combine them by using a tensor product to produce the indefinite superposition state. Such operations result in a complete set of possible states (i.e., $|000\rangle,|100\rangle \ldots .|111\rangle)$. However, because of our assumption that either all or none of the associates are activated, the only states that can occur are $|1\rangle \otimes|1\rangle \otimes|1\rangle=|111\rangle$ and $|0\rangle \otimes|0\rangle \otimes|0\rangle=|000\rangle$, respectively. Given this restriction, the activation state of the whole system can be written as an entangled state:

$\left|\psi_{t}\right\rangle=\sqrt{p_{0}}|000\rangle+\sqrt{p_{1}}|111\rangle$.

Equation 11 describes an entangled state representing the target in its indefinite form, where $p_{0}$ and $p_{1}$ represent the probabilities that none $(|000\rangle)$ or that all $(|111\rangle)$ of the associates are activated, respectively. We can extract values for $p_{0}$ and $p_{1}$ by making the following observations using the law of total probability:

$p_{0}=\bar{p}_{\mathrm{a} 1} \bar{p}_{\mathrm{a} 2} \bar{p}_{\mathrm{a} 3}$,
Fig. 7 System of related associates shown in Table 7, represented as a set of three qubits
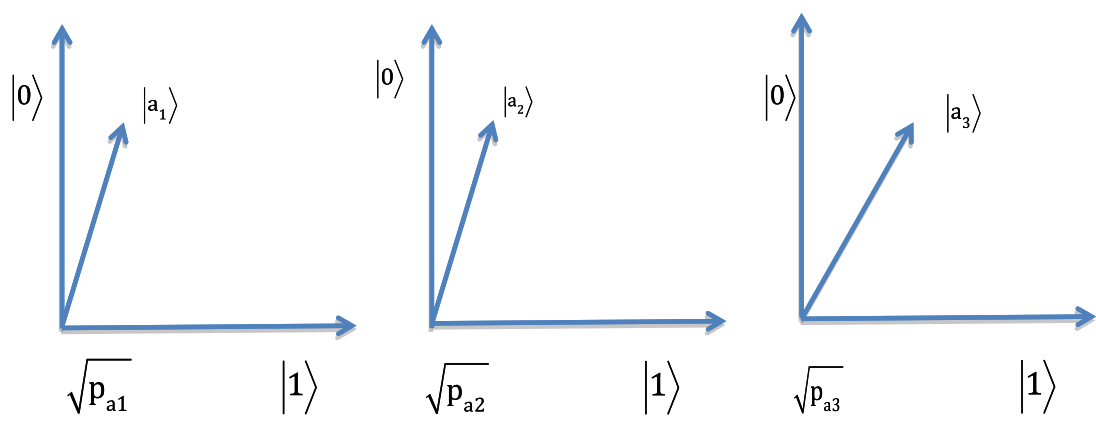
and therefore,

$p_{1}=1-p_{0}=1-\bar{p}_{a 1} \bar{p}_{a 2} \bar{p}_{a 3}$.

Given this activation assumption, the remaining probability mass contributes to primed target strength as a whole. Consequently,

$p_{1}=1-\left(1-p_{a 1}\right)\left(1-p_{a 2}\right)\left(1-p_{a 3}\right)$,

so in Table 7 ,

$p_{1}=1-(1-.167)(1-.167)(1-.333)=.537$.

Equation 14 can be generalized for targets having any number of associates, and we identify it and its assumptions as the "entanglement model," or $\mathrm{EMS}_{t}$, for predicting the target-priming effects described by $S_{t^{\prime}}$ (Eq. 4). $\mathrm{EMS}_{t^{\prime}}$ is successful to the extent that it correlates with $S_{t^{\prime}}$, and to evaluate the model, we used Eq. 14 to compute the primed target strength for all targets studied in the standard conditions in the extralist-cuing database. The correlation between EMS $_{t^{\prime}}$ and $S_{t^{\prime}}$ is $r=.987, n=2,803$, and $R^{2}=97.4 \%$, so nearly all of the variance of our intuitive descriptive statistic is predicted by the entanglement model. When we enter $\mathrm{EMS}_{t^{\prime}}$ in place of $S_{t^{\prime}}$ along with $S_{q t}, S_{t q}$, and $S_{q d}$ as predictors of recall in a multiple regression analysis, the results are nearly identical $\left[F(4,2798)=390.97, M S_{\text {res }}=.043, R=.599, R^{2}=.358\right]$, as compared to when $S_{t^{\prime}}$ is used (Table 3). The correlations with probability of recall for $\mathrm{EMS}_{t^{\prime}}$ and $S_{t^{\prime}}$ are $r=.264$ and .256 , respectively. Because the results are so similar, Eq. 1 predicts that the two methods for computing target activation will have similar correlations with recall and similar predictions of mean recall, and this is the case. Recall probability is correlated $r=.567$ for both the $\mathrm{EMS}_{t^{\prime}}$ and $S_{t^{\prime}}$ measures, and mean recall is $.392(S D=.159)$ and $.390(S D=.162)$. The mean differences between $S_{t^{\prime}}$ and $\mathrm{EMS}_{t^{\prime}}$ tend to be very small, averaging .01, because $\mathrm{EMS}_{t}$, tends to predict lower values of target activation than does $S_{t^{\prime}}$ for a few targets that have only several associates (as in the example calculation in Table 7).

The entanglement model is successful because it captures the effects of links among the target's associates, as well as the intuitive measure $S_{t^{\prime}}$. According to the model, the appearance of the target during study puts it into an indefinite superposition state in which its associates are simultaneously activated and not activated in the experimental context until the target's representation enters working memory, where it is observed and can be rehearsed or its properties can be judged. As soon as the target appears in working memory, subjects become consciously aware of its existence in the experimental setting, and the superposition state collapses to either a nonprimed or a primed state. This interpretation assumes that a minuscule amount of time elapses between when a word appears on the screen and when it is observed. During this interval, the target is retrieved from the semantic network as a superposition state. When the superposition collapses to its primed state, priming effects emerge, and encoding a target in this state in working memory links it to the contextual cues available in the setting. In this approach, encoding processes applied in accordance with the study instructions operate on the target's representation in working memory, regardless of the value of its primed state in the system. When all of the target's associates are activated, a primed representation of $T$ is loaded into working memory, and when none are activated, an unprimed representation of $T$ is loaded into this system.

Another similarity that makes the comparison interesting between physical and semantic states of entanglement concerns the effects of semantic context. Quantum research has indicated that superposition states are not permanent. Particles and other objects existing in entangled states suddenly collapse to one or the other state when measured (Laloe, 2001). Analogously, seeing a target in semantic isolation locates it in its semantic neighborhood and creates a superposition state in the experimental setting that collapses to activating none or all of its associates when a subject becomes consciously aware of it in working memory. In contrast, when the target is seen in the context provided by a semantically related word, neighborhood density effects are not apparent, because the target's meaning is specified by the context. Seeing a semantically related cue-target pairing creates an entangled state in which the target's associates are or are not activated, but this state immediately collapses to the nonactivated state when this representation reaches working memory. The target's superposition state prior to appearing in working memory is the same as when it is seen in isolation, but when it is seen in an appropriate semantic context, the superposition state collapses to a nonactivated state when observed in working memory. Although derived from an unusual source for the field of memory, the entanglement model fares better as a model of target activation effects than does spreading activation, because it is based on a parallel activation process that is sensitive to environmental context.

\section{General discussion}

Both experimental and correlational findings have shown how the semantic network contributes to event memory in tasks involving a semantically isolated target and a cue that is related to the target, or that is the target in a physical sense. The magnitude of the network contribution based on variance explained differs across tasks, depending on the semantic context during encoding, context awareness during testing, and the nature of the cue. Importantly, in extralist cuing the network contributes more to recall than does study time or how the target is encoded during study. Recall in this 
task is affected by both semantic and episodic information, and the semantic information is critical, because recall is based on a test cue that is both absent during study and semantically related to the target. The network provides semantic information during study that defines the meaning of an isolated word event, and during testing it provides information that guides the search for this event. During both study and test, familiar words act as reminding cues that reliably and quickly activate the prior semantic contexts of these words. In what follows, we consider four general and partially overlapping conclusions.

\section{The semantic network}

The first conclusion is that a semantic network exists in memory as a complex system of words and links that functions as a repository of word knowledge. Words are not randomly connected in this system (Collins \& Loftus, 1975; Steyvers \& Tenenbaum, 2005). Rather, they cluster around similar experiences involving thousands of direct links and tens of thousands of indirect links that may provide shortcuts across the domain (Watts \& Strogatz, 1998). This system continually interacts with familiar words, objects, and events by automatically activating related words. Automatic parallel activation provides the informational bases for comprehension, priming, and encoding, as well as for search, recall, and recognition. Episodic encoding and retrieval depend to varying degrees on the semantic network to provide the word knowledge needed in each of these processes, and more specifically, the findings show that measures based on the network are correlated with memory performance in several event memory tasks. Of the four key measures taken on the network, word recognition relies only on target activation; intralist cuing relies only on cue-totarget strength; and extralist cuing depends on all four measures. Taken together, the findings provide strong evidence for the hypothesis that words are represented and processed in a vast dynamic semantic storage system that contributes to and that can be used to predict the recall and recognition of episodic events.

\section{Free association and semantic context}

The second general conclusion is that free association to thousands of words can be used to generate a semantic network that measures what related words are likely to come to mind when experiencing a word-measures that are reliable and valid, and that are sensitive to semantic context. This method is reliable because renorming the same word tends to produce the same associates for even weaker associates, and it is valid because measures taken from this network predict behavior in various memory tasks. Finally, the network is sensitive to context, because the same word takes on different shades of meaning when influenced by different cues. For example, in our free-association norms, food is produced as a response by $324 / 5,018$ different cues, and its meaning varies for each cue. Each cue that produces food as an associate also produces related words that vary substantially across cues, so that food means something different in the context of different words. Both Dinner and Hamburger produce food as an associate, but otherwise their associates are completely different, indicating that food activates different semantic senses in the contexts provided by Dinner and Hamburger. Free-association norms capture context-driven differences in meaning, because a word with exactly the same name appears among different associates for each cue that generates it in free association. We suggest that the semantic network generated via free association is highly sensitive to semantic context differences because the cues being normed activate not just a single word, but an array of related words out of which one or more words can be selected.

\section{Activation, search, and memory task}

The third conclusion concerns the distinction between the activation associated with priming effects and search. The most advanced memory models of recall generally do not explicitly incorporate activation in the modeling, because the research is based on the free recall of unrelated words, defined as words that are not directly associated (e.g., Gillund \& Shiffrin, 1984; Shiffrin \& Steyvers, 1997). The study phases in free-recall and extralist-cuing tasks are essentially identical, so it is reasonable to assume that the target words are loaded into working memory as either primed or not primed, but the testing phases are different. In free recall, subjects are asked to recall all list words in any order, whereas in cued recall, a semantically related cue is used to prompt the recall of each one. In extralist cuing, the semantic cue provides access to an array of response alternatives that include the target, whereas in free recall the first word recalled is cued by context, and the remaining words are cued through a combination of context and episodic associations between the words produced by rehearsal during study (Raaijmakers, \& Shiffrin, 1981). In the free recall of unrelated words, what is activated in the semantic network serves no apparent purpose. There is no reason to believe that the activation of related words by the target or the cue might be important, so the priming literature and the concept of activation have been largely and reasonably ignored in modeling free recall.

The results of three experiments that compared the two tasks directly, using the same materials and study conditions, justified this position (Nelson, Akirmak, \& Malmberg, in preparation). These experiments showed that neighborhood density and the resulting priming effects are strong in extralist 
cuing and absent in free recall. Although both tasks involve search, the sets that are being searched are different: In free recall, memory for the study list is searched using a recalled word as a cue for recalling another word from the study list, on the basis of an episodically created link (Raaijmakers \& Shiffrin, 1981). In contrast, in extralist cuing, the cue activates its associates in the semantic network, and search is limited to this set. The network becomes involved in recall when a test cue is semantically linked to its prospective target, and the task comparison suggests that target-priming effects are contingent on recovering the target from its storage in this network: Target-priming effects may be observed only when searching the network that created these effects in the first place.

These findings suggest that the network plays a critical role in recall that is cued by a meaningfully related word, but no role whatsoever when the recall of one list word is cued by an unrelated word. They also indicate that, when the semantic system is utilized, effects of related words can be apparent during both study and test. Related words are not physically presented, so some brain mechanism must be providing them, and the findings here and in the priming literature suggest that implicit activation is that mechanism. The evidence reviewed here suggests that the semantic memory system supports the recognition and recall of episodic events by activating related words that serve as the basis for comprehension and search processes in some, but not all, episodic memory tasks. In the extralist-cuing task, the simultaneous activation of the target's associates during the study phase provides the semantic context for a word seen in isolation, and during testing, the cue activates a set of potential responses that is likely to include the target and that initiates a search for the target within this set. Activation plays a role in both phases, but the study phase is largely concerned with comprehension and following the study instructions. The key network variable in this phase is neighborhood density - specifically, its influence on target priming. In contrast, the test phase is largely concerned with search, and the key network variables are cue-to-target strength, cue-to-distractor strength, and target-to-cue strength. Variables associated with the semantic network contribute in different ways to both phases of extralist cuing, and in different ways to recognition and intralist cuing, suggesting that the network responds dynamically to the contexts provided by different ways of measuring memory. Semantic memory is a fast-acting dynamic system that reminds us about what we know. To return to the beginning, our findings indicate that the electric company is paid because check, envelope, and mailbox activate related words that allow mailbox to succeed as a cue by reminding us to pay the bill.

\section{Pier3 and entanglement}

Equation 1 provides an algorithm for predicting extralist cuing that evolved from the Pier framework, and we refer to it as
Pier3. Pier3 computations are derived from measures made on the semantic network that are combined in a ratio rule that predicts the contribution of the semantic system to probability of recall. Pier3's core rationale is motivated by the SAM model ("search of associative memory"; Raaijmakers \& Shiffrin, 1981), but it is incomplete as a SAM model, because it does not incorporate the effects of environmental context or of encoding operations in calculating its predictions. In addition, it differs from SAM in that search is based on a semantic network of links acquired in interactions with the world, as opposed to an episodic network of associative links established during recent study. In addition, Pier3 makes probabilistic predictions for specific cue-target pairs. By computing mean predicted recall and the standard deviation for pairs linked to a specific semantic manipulation (e.g., neighborhood density), it predicts the contribution of the semantic network to recall in extralist-cuing experiments. In extralist cuing, once a list is prepared, it is possible to compute a recall prediction for each cue-target pair, and by averaging over the pairs in a given condition, it is possible to predict recall for that condition. Finally, Pier3 also differs from SAM in how it aggregates links among related words, but despite the differences, both approaches assume that recall is based on searching memory for recently encoded information. Given this commonality, it might be possible to develop a SAM model for extralist cuing that incorporates simulations of episodic encoding processes using predictions based on semantic relationships. In Bayesian terms, Pier3 predictions could serve as the prior probability, and a to-be-developed measure of the contribution of episodic memory could serve as the posterior probability.

In Pier3, the test cue initiates a search of the semantic network, and this search is facilitated when the cue and its target are more strongly linked relative to all of the information relevant to its selection, including the error introduced by the cue's distractors. Recall is more likely when the cue-totarget and target-to-cue strengths are high, and when cue-todistractor strength is weak. It is also more likely when target priming is higher, when the target is studied longer, and when it is studied with a more effective procedure. Pier3 assumes that both target priming and encoding strengthen its representation in the semantic network and that this elevation decays over time unless refreshed.

The target-priming effect is in many ways a most interesting phenomenon, because it is founded on links among the target's associates. Classic spreading-activation theory predicts that this result arises because activation in the target's network returns to the target, as opposed to spreading outside its neighborhood. This interpretation, however, is countered by evidence indicating that target-priming effects are independent of the presence of associate-to-target links. Target-priming effects do not appear to arise from a sequential activation process, but from a parallel process 
that simultaneously activates the target's neighborhood. Words exist in a tangled network of associative/semantic links, and when context fails to provide a specific semantic sense, the network activates and temporarily encodes its most immediate senses. The entanglement model captures this process effectively because it is based on an indefinite superposition state that is sensitive to context, represented in the probabilities that none and all of a target's associates are activated. The model assumes that the physical appearance of a word puts it into a superposition state that collapses to a specific sense when the word reaches working memory, and an individual becomes aware of its existence. The target becomes entangled with its associates, and such entanglement occurs when the target and its associates are simultaneously activated. When applied to the extralist-cuing database, the model explains $97.4 \%$ of the variance of the intuitive measure of neighborhood density. Although rarely used in psychological research, this result indicates that the superposition assumption can be productively used to study difficult problems involving indefinite semantic states and, possibly, other indefinite psychological states involving uncertainty (Busemeyer \& Bruza, 2012).

Author note The authors thank Lili Sahakyan and the reviewers and editor for their exceptionally helpful suggestions. We also offer heartfelt thanks to all of the students who worked on this project over its 30year existence. This project was supported in part by Australian Research Council Discovery grant DP1094974.

\section{References}

Aerts, D. (2009). Quantum structure in cognition. Journal of Mathematical Psychology, 53, 314-348.

Anderson, J. R. (1983). A spreading activation theory of memory. Journal of Verbal Learning and Verbal Behavior, 22, 261-295. doi:10.1016/S0022-5371(83)90201-3

Barabási, A.-L. (2002). Linked: The new science of networks. Cambridge, MA: Perseus.

Battig, W. F., \& Montague, W. E. (1969). Category norms of verbal items in 56 categories: A replication and extension of the Connecticut category norms. Journal of Experimental Psychology, 80(3, Pt. 2), 1-46. doi:10.1037/h0027577

Borge-Holthoefer, J., \& Arenas, A. (2010). Semantic networks: structure and dynamics. Entropy, 12, 1264-1302.

Brainerd, C. J., Yang, Y., Reyna, V. F., Howe, M. L., \& Mills, B. A. (2008). Semantic processing in "associative" false memory. Psychonomic Bulletin \& Review, 15, 1035-1053. doi:10.3758/ PBR.15.6.1035

Bruza, P., Kitto, K., Nelson, D., \& McEvoy, C. (2009). Is there something quantum-like about the human mental lexicon? Journal of Mathematical Psychology, 53, 362-377. doi:10.1016/ j.jmp.2009.04.004

Busemeyer, J. R., \& Bruza, P. (2012). Quantum models of cognition and decision making. New York, NY: Cambridge University Press.

Busemeyer, J. R., Pothos, E., Franco, R., \& Trueblood, J. (2011). A quantum theoretical explanation for probability judgment errors. Psychological Review, 118, 193-218.
Chappell, M., \& Humphreys, M. S. (1994). An auto-associative neural network for sparse representations: Analysis and application to models of recognition and cued recall. Psychological Review, 101, 103-128.

Collins, A. M., \& Loftus, E. F. (1975). A spreading-activation theory of semantic processing. Psychological Review, 82, 407-428. doi:10.1037/0033-295X.82.6.407

Cramer, P. (1968). Word association. New York, NY: Academic Press.

De Deyne, S., \& Storms, G. (2008a). Word associations: Network and semantic properties. Behavior Research Methods, 40, 213-231. doi:10.3758/BRM.40.1.213

De Deyne, S., \& Storms, G. (2008b). Word associations: Norms for 1,424 Dutch words in a continuous task. Behavior Research Methods, 40, 198-205.

Deese, J. (1965). The structure of associations in language and thought. Baltimore, MD: Johns Hopkins Press.

Fisher, S. L., \& Nelson, D. L. (2006). Recursive reminding: Effects of repetition, printed frequency, connectivity, and set size on recognition and judgments of frequency. Memory \& Cognition, 34, 295-303. doi:10.3758/BF03193408

Gabora, L., \& Aerts, D. (2002). Contextualizing concepts using a mathematical generalization of the quantum formalism. Journal of Experimental and Theoretical Artificial Intelligence, 14, 327-358.

Galea, D., Bruza, P. D., Kitto, K., Nelson, D., \& McEvoy, C. L. (2011). Modeling the activation of human memory: The spreading activation, spooky action at a distance, and the entanglement models compared. In D. Song \& M. Melucci (Eds.), Proceedings of the 5th Quantum Interaction International Symposium (QI-2011) vol. 7052 (pp. 149-160). Berlin: Springer.

Gee, N. (1997). Implicit memory and word ambiguity. Journal of Memory and Language, 36, 253-275.

Gillund, G., \& Shiffrin, R. M. (1984). A retrieval model for both recognition and recall. Psychological Review, 91, 1-67. doi:10.1037/ 0033-295X.91.1.1

Goldman, M. S. (1999). Risk for substance abuse: Memory as a common etiological pathway. Psychological Science, 10, 196-199.

Goodmon, L. B., \& Nelson, D. L. (2004). Strengthening the activation of unconsciously activated memories. Memory \& Cognition, 32, 804-818. doi:10.3758/BF03195870

Greenstein, G., \& Zajonc, A. G. (2006). The quantum challenge: Modern research on the foundations of quantum mechanics (2nd ed.). Sudbury, MA: Jones \& Bartlette.

Griffiths, T. L., Steyvers, M., \& Tenenbaum, J. B. (2007). Topics in semantic representation. Psychological Review, 114, 211-244. doi:10.1037/0033-295X.114.2.211

Hills, T. T., Maouene, M., Riordan, B., \& Smith, L. (2010). The associative structure of language: Contextual diversity in early word learning. Journal of Memory and Language, 63, 259-273.

Holley, P. E., \& McEvoy, C. L. (1996). Aging and inhibition of unconsciously processed information: No apparent deficit. Applied Cognitive Psychology, 10, 241-256.

Humphreys, M. S., Bain, J. D., \& Pike, R. (1989). Different ways to cue a coherent memory system: A theory for episodic, semantic, and procedural tasks. Psychological Review, 96, 208-233. doi:10.1037/0033-295X.96.2.208

Humphreys, M. S., Tehan, G., O'Shea, A., \& Bolland, S. W. (2000). Target similarity effects: Support for the parallel distributed processing assumptions. Memory \& Cognition, 28, 798-811.

Hutchison, K. A. (2003). Is semantic priming due to association strength or feature overlap? A microanalytic review. Psychonomic Bulletin \& Review, 10, 785-813. doi:10.3758/BF03196544

Isham, C. J. (1995). Lectures on quantum theory. London, UK: Imperial College Press.

Jeong, H., Tombor, B., Albert, R., Oltvai, Z. N., \& Barabási, A.-L. (2000). The large-scale organization of metabolic networks. Nature, 407, 651-654. 
Kintsch, W. (1988). The role of knowledge in discourse comprehension: a construction-integration model. Psychological Review, 95, 163-182. doi:10.1037/0033-295X.95.2.163

Laloe, F. (2001). Do we really understand quantum mechanics? Strange correlations, paradoxes and theorems. American Journal of Physics, 69, 655-701.

MacRae, K., Seidenberg, M. S., \& McNorgen, C. (2005). Semantic feature production norms for a large set of living and nonliving things. Behavior Research Methods, 37, 547-559.

Maki, W. S., \& Buchanan, E. (2008). Latent structure in measures of associative, semantic, and thematic knowledge. Psychonomic Bulletin \& Review, 15, 598-603. doi:10.3758/PBR.15.3.598

Malmberg, K. J., Steyvers, M., Stephens, J. D., \& Shiffrin, R. M. (2002). Feature frequency effects in recognition memory. Memory \& Cognition, 30, 607-613. doi:10.3758/BF03194962

Maudlin, T. (1994). Quantum non-locality and relativity: Metaphysical intimations of modern physics. Oxford, UK: Oxford University Press.

Monaco, J. D., Abbott, L. F., \& Kahana, M. J. (2008). Lexico-semantic structure and the word frequency effect in recognition memory. Learning and Memory, 14, 204-213.

Moreno, J. L. (1934). Who shall survive? Beacon NY: Beacon House.

Myers, J. L., \& Well, A. D. (1995). Research design and statistical analysis. Hillsdale: Erlbaum.

Nelson, D. L., Bajo, M.-T., \& Casanueva, D. (1985). Prior knowledge and memory: The influence of natural category size as a function of intention and distraction. Journal of Experimental Psychology: Learning, Memory, and Cognition, 11, 94-105. doi:10.1037/ 0278-7393.11.1.94

Nelson, D. L., Bajo, M.-T., McEvoy, C. L., \& Schreiber, T. A. (1989). Prior knowledge: The effects of natural category size on memory for implicitly encoded concepts. Journal of Experimental Psychology: Learning, Memory, and Cognition, 15, 957-967. doi: $10.1037 / 0278-7393.15 .5 .957$

Nelson, D. L., Bennett, D. J., Gee, N. R., Schreiber, T. A., \& McKinney, V. M. (1993). Implicit memory: Effects of network size and interconnectivity on cued recall. Journal of Experimental Psychology: Learning, Memory, and Cognition, 19, 747-764. doi:10.1037/0278-7393.19.4.747

Nelson, D. L., \& Castano, D. (1984). Mental representations for pictures and words: same or different? The American Journal of Psychology, 97, 1-15.

Nelson, D. L., Dyrdal, G. M., \& Goodmon, L. B. (2005). What is preexisting strength? Predicting free association probabilities, similarity ratings, and cued recall probabilities. Psychonomic Bulletin \& Review, 12, 711-719.

Nelson, D. L., Fisher, S. L., \& Akirmak, U. (2007a). How implicitly activated and explicitly acquired knowledge contribute to the effectiveness of retrieval cues. Memory \& Cognition, 35, 18921904. doi:10.3758/BF03192923

Nelson, D. L., Gee, N. R., \& Schreiber, T. A. (1992a). Sentence encoding and implicitly activated memories. Memory \& Cognition, 20, 643-654.

Nelson, D. L., \& Goodmon, L. B. (2002). Experiencing a word can prime its accessibility and its associative connections to related words. Memory \& Cognition, 30, 380-398. doi:10.3758/ BF03194939

Nelson, D. L., \& Goodmon, L. B. (2003). Disrupting attention: The need for retrieval cues in working memory theories. Memory \& Cognition, 31, 65-76. doi:10.3758/BF03196083

Nelson, D. L., Goodmon, L. B., \& Akirmak, U. (2007b). Implicitly activated memories are associated to general context cues. Memory \& Cognition, 35, 1878-1891. doi:10.3758/BF03192922

Nelson, D. L., Goodmon, L. B., \& Ceo, D. (2007c). How does delayed testing reduce effects of implicit memory: Context infusion or cuing with context? Memory \& Cognition, 35, 1014-1023. doi:10.3758/BF03193474

Nelson, D. L., \& McEvoy, C. L. (1979). Encoding context and set size. Journal of Experimental Psychology: Human Learning and Memory, 5, 292-314.

Nelson, D. L., McEvoy, C. L., \& Dennis, S. (2000). What is free association and what does it measure? Memory \& Cognition, 28, 887-899. doi:10.3758/BF03209337

Nelson, D. L., McEvoy, C. L., \& Pointer, L. (2003). Spreading activation or spooky action at a distance? Journal of Experimental Psychology: Learning, Memory, and Cognition, 29, 42-51. doi:10.1037/0278-7393.29.1.42

Nelson, D. L., McEvoy, C. L., \& Schreiber, T. A. (2004). The University of South Florida free association, rhyme, and word fragment norms. Behavior Research Methods, Instruments, \& Computers, 36, 402-407. doi:10.3758/BF03195588

Nelson, D. L., McKinney, V. M., Gee, N. R., \& Janczura, G. A. (1998). Interpreting the influence of implicitly activated memories on recall and recognition. Psychological Review, 105, 299-324.

Nelson, D. L., Schreiber, T. A., \& McEvoy, C. L. (1992b). Processing implicit and explicit representations. Psychological Review, 99, 322-348.

Nelson, D. L., Walling, J. R., \& McEvoy, C. L. (1979). Doubts about depth. Journal of Experimental Psychology: Human Learning and Memory, 5, 24-44.

Nelson, D. L., \& Zhang, N. (2000). The ties that bind what is known to the recall of what is new. Psychonomic Bulletin \& Review, 7, 604 617. doi:10.3758/BF03212998

Nelson, D. L., Zhang, N., \& McKinney, V. M. (2001). The ties that bind what is known to the recognition of what is new. Journal of Experimental Psychology: Learning, Memory, and Cognition, 27, 1147-1159. doi:10.1037/0278-7393.27.5.1147

Newman, M. E. J. (2003). The structure and function of complex networks. SIAM Review, 45, 167-256.

Palla, G., Derényi, I., Farkas, I., \& Vicsek, T. (2005). Uncovering the overlapping community structure of complex networks in nature and society. Nature, 435, 814-818.

Pothos, E., \& Busemeyer, J. R. (2009). A quantum probability explanation for violations of "rational" decision theory. Proceedings of the Royal Society B, 276, 193-218.

Raaijmakers, J. G. W., \& Shiffrin, R. M. (1981). Search of associative memory. Psychological Review, 88, 93-134. doi:10.1037/0033295X.88.2.93

Reder, L. M., \& Anderson, J. R. (1980). A partial resolution of the paradox of interference: The role of integrating knowledge. Cognitive Psychology, 12, 447-472.

Reich, R. R., \& Goldman, M. (2005). Exploring the alcohol expectancy network: The utility of free associates. Psychology of Addictive Behaviors, 19, 317-325.

Sahakyan, L., \& Goodmon, L. B. (2010). Theoretical implications of extralist probes for directed forgetting. Journal of Experimental Psychology: Learning, Memory, and Cognition, 36, 920-937.

Shiffrin, R. M., \& Steyvers, M. (1997). A model for recognition memory: REM - retrieving effectively from memory. Psychonomic Bulletin \& Review, 4, 145-166. doi:10.3758/BF03209391

Stacy, A. W. (1997). Memory activation and expectancy as prospective predictors of alcohol and marijuana use. Journal of Abnormal Psychology, 106, 61-73.

Steyvers, M., Shiffrin, R. M., \& Nelson, D. L. (2005). Word association spaces for predicting semantic similarity effects in episodic memory. In A. F. Healy (Ed.), Experimental cognitive psychology and its applications (pp. 237-249). Washington, DC: American Psychological Association.

Steyvers, M., \& Tenenbaum, J. B. (2005). The large-scale structure of semantic networks: Statistical analyses and a model of 
semantic growth. Cognitive Science, 29, 41-78. doi:10.1207/ s15516709 $\operatorname{cog} 29013$

Strogatz, S. H. (2003). SYNC: The emerging science of spontaneous order. New York, NY: Hyperion Books.

Tulving, E., \& Thomson, D. M. (1973). Encoding specificity and retrieval processes in episodic memory. Psychological Review, 80, 352-373. doi:10.1037/h0020071
Turvey, M. T., \& Moreno, M. A. (2006). Physical metaphors for the mental lexicon. Mental Lexicon, 1, 7-33.

Watts, D. J., \& Strogatz, S. H. (1998). Collective dynamics of "smallworld" networks. Nature, 393, 440-442.

Zeelenberg, R., Shiffrin, R. M., \& Raaijmakers, J. G. W. (1999). Priming in a free association task as a function of association directionality. Memory \& Cognition, 27, 956-961. doi:10.3758/BF03201226 